SUPPLEMENT

\title{
Heading in football. Part 1: Development of biomechanical methods to investigate head response
}

\author{
N Shewchenko, C Withnall, M Keown, R Gittens, J Dvorak
}

Br J Sports Med 2005;39(Suppl I):i10-i25. doi: 10.1136/bjsm.2005.019034

See end of article for authors' affiliations

\section{Correspondence to:} N Shewchenko, Biokinetics and Associates Ltd, Ontario, Canada: shewchenko@biokinetics. com

\begin{abstract}
Objectives: There has been growing controversy regarding long term effects of repeated low severity head impacts such as when heading a football. However, there are few scientific data substantiating these concerns in terms of the biomechanical head response to impact. The present study aimed to develop a research methodology to investigate the biomechanical response of human subjects during intentional heading and identify strategies for reducing head impact severity.

Methods: A controlled laboratory study was carried out with seven active football players, aged 20-23 and of average stature and weight. The subjects were fitted with photographic targets for kinematic analysis and instrumented to measure head linear/angular accelerations and neck muscle activity. Balls were delivered at two speeds $(6 \mathrm{~m} / \mathrm{s}$ and $8 \mathrm{~m} / \mathrm{s})$ as the subjects executed several specific forward heading manoeuvres in the standing position. Heading speeds up to $11 \mathrm{~m} / \mathrm{s}$ were seen when the head closing speed was considered. One subject demonstrating averaged flexion-extension muscle activity phased with head acceleration data and upper torso kinematics was used to validate a biofidelic 50th percentile human model with a detailed head and neck. The model was exercised under ball incoming speeds of 6-7 m/s with parameter variations including torso/head alignment, neck muscle tensing, and follow through. The model output was subsequently compared with additional laboratory tests with football players $(n=3)$. Additional heading scenarios were investigated including follow through, non-active ball impact, and noncontact events. Subject and model head responses were evaluated with peak linear and rotational accelerations and maximum incremental head impact power.

Results: Modelling of neck muscle tensing predicted lower head accelerations and higher neck loads whereas volunteer head acceleration reductions were not consistent. Modelling of head-torso alignment predicted a modest reduction in volunteer head accelerations. Exaggerated follow through while heading reduced volunteer head accelerations modestly.

Conclusion: Biomechanical methods were developed to measure head impact response. Changing the biomechanics of currently accepted heading techniques will have inconsistent benefits towards the reduction of head loading. Furthermore, mathematical modelling suggested an increased risk of neck loads with one alternative technique. No consistent recommendations can be made on the basis of the current study for altering heading techniques to reduce impact severity.
\end{abstract}

$\mathrm{F}$ otball, or soccer in North America, is unique among sports in that the head is intentionally used to contact and guide the ball as part of offensive and defensive game play.

\section{BACKGROUND AND OBJECTIVES Head injuries in football}

The incidence of head injuries has been found to be infrequent relative to all other injuries experienced in football and those resulting from frequent heading of the ball are even less apparent. ${ }^{1-6}$ With the increasing popularity of football-expanding at a rate of $10 \%$ per year with over 250 million players registered worldwide in 2003 (www.fifa. com) - the potential consequences and resulting long term sequelae of heading are being questioned. Published studies suggesting a potential relation between heading and neurophysiological and neuropsychological dysfunction have been regularly referenced by the media despite the lack of conclusive data.

Recent studies on the effects of heading have helped quantify the incidence of head injuries but much controversy remains with respect to the long term effects. A series of studies of active and retired players showed cognitive deficits to be associated with repeated headings. ${ }^{58}$ The authors found no statistically significant difference between the electroencephalographic responses of inexperienced headers and non-headers. Cerebral computed tomography scans of former players revealed cerebral atrophy and neuropsychological examinations showed mild to severe cognitive deficits. The results of these studies have been quoted extensively in the media when justifying the need for preventive measures such as limiting the exposure of headings with youth or in the endorsement of football headgear. However, the studies were found to be flawed due to poor methodology including the lack of controls and pre-injury data, selection bias, failure to control acute injuries, and lack of blind observers. ${ }^{9}$

Similar observations as above were obtained in a study of amateur and professional players indicating that both concussive injury and heading were associated with diminished cognitive function. ${ }^{10-12}$ Links were found between the number of concussions and test performance on memory and planning and between the number of headers and neuropsychological test performance for professional players. However, a comment was made that heading may not be associated with cognitive impairment. ${ }^{13}$ In a study of youth

Abbreviations: $C T B I$, chronic traumatic brain injury; $E M G$ electromyography; HIP, Head Impact Power (index); MTBI, mild traumatic brain injury; MVC, mean voluntary contraction; NFL, National Football League; RMS, root mean square; TBI, traumatic brain injury; WSTC, Wayne State Tolerance Curve 
players (average age 11.5 years) cognitive performance was assessed in relation to heading. ${ }^{14}$ Abnormalities were found including impaired learning of new words and experiencing headaches ( $49 \%$ of cases).

In a study of active collegiate players that included control groups, no evidence of diminished neuropsychological performance was found..$^{15}$ Other studies and reviews of chronic brain injury indicate that the correlation with heading is inconclusive. ${ }^{15-20}$ Some of the findings from earlier studies may also have limited applicability to modern day players because they were based on former professional football players using old generation balls with higher mass when wet. A recent observation in the available research indicates that it is not known whether a relation exists between sub-concussive headings and chronic cognitive impairment.' It was further suggested that heading is unlikely to cause these injuries, and that although concussive injuries can occur from head to head impacts they are unlikely to contribute to cumulative injuries.

In terms of the incidence of head injuries, head impacts during heading duels have been found to be the main mechanism of injury. ${ }^{21}$ Elbow to head impacts were found to be the most frequent $(41 \%)$, followed by head to head impacts (32\%). Although not all impacts can be reduced, the effects of upper extremity impacts can be mitigated with more forceful implementation of game play rules.

The documented low incidence of injurious ball to head impacts $^{21}$ and difficulty in diagnosing the injuries, whether they are mild, acute or chronic in nature, also questions the need for analysis of headings. Despite the lack of scientific data, the concerns related to this type of injury mechanism have already resulted in changes to the game at the professional and non-professional levels. The use of headgear has become more prominent as well as implementation of changes in coaching methods, especially for youths. There is therefore a need to understand the threat to the players and the impact biomechanics for establishing reduction measures to manage injury risks, if required.

\section{Implications of heading techniques}

Guidelines and techniques for heading currently exist for improving the skills of players. The techniques have also been observed to alter the head response under impact, and hence, injury potential. ${ }^{81722}$ Coaches and trainers, through experience, have developed specific guidelines to reduce head loading and discomfort while improving heading effectiveness. However, due to the lack of scientific justification at this time further research into heading biomechanics is necessary. Furthermore, biomechanical studies can help identify physiological parameters related to head response which could lead to a better understanding of external influences on head impact severity, such as the ball. Recent biomechanical studies have started to provide insight into the specific aspects of heading kinematics and physiological response, ${ }^{23-25}$ but remain to provide recommendations for mitigating head impact severity.

\section{Biomechanical analysis techniques}

Biomechanical analysis of heading techniques will provide valuable insight into the causes and factors contributing to head loading. Perhaps more importantly, it can form the basis for preventive measures through the modification of heading techniques for reducing head loading and the related potential for injury. Biomechanical analysis techniques reported in literature include both empirical and analytical approaches. Empirical methods measure the body motions (kinematics) and forces (kinetics) exerted on the body as opposed to analytical methods which predict bodily responses by replicating the impact dynamics.
Kinematic analysis techniques provide information on body motions. The techniques generally consist of cinematography and motion tracking systems offering two or three dimensional information on body segment translation, rotation, velocity and to a limited extent, acceleration. In a review of published biomechanical studies, it was noted that ball impact forces determined through motion analysis and cinematography techniques are prone to inaccuracies. ${ }^{24}$ This can likely be attributed to the poor bandwidth and resolution of the systems to resolve the short duration accelerations $(<30 \mathrm{~ms})$ experienced in this type of impact environment and poor characterisation of the energy losses.

The kinetic responses of interest for the current study included peak head acceleration and power transfer since these are related to measures of injury assessment. Until such time when the assessment of chronic traumatic brain injury (CTBI) is fully defined, the injury measures in this study must only be considered as relative measures of impact severity. Kinetic measurement techniques include linear accelerometry for direct measure of head impact response..$^{23-29}$ Measurement of linear acceleration is typically accomplished with the sensor mounted onto the head for direct measurement in one axis or combined with multiple units to quantify accelerations in two or three dimensions. Accelerometry techniques are the basis of biomechanical analysis, but many current studies employing these techniques are limited with respect to $(a)$ the variety of heading techniques used by the test subjects and $(b)$ the details of the measurements taken in the studies preventing complete characterisation of the event. More recent studies have provided greater insight into head acceleration responses during heading but have failed to elaborate on the consequences of using different heading techniques. ${ }^{25}$

Angular accelerometry techniques are also included in kinetic analysis methods and have been used to study head responses in heading due to its implication with diffuse brain injuries. ${ }^{23}{ }^{25}$ Angular accelerations about one or more axes are derived from multiple linear acceleration measurements and require careful implementation to ensure accuracy..$^{23} 250$ Existing single axis transducers measure angular acceleration or velocity directly but operate within specific frequency ranges limiting their usefulness. ${ }^{30}$

In alternative kinetic approaches, the forces created during ball to head impacts have been measured directly to assess the influence of heading type and approach. ${ }^{31}$ Measurements were taken with an array of gel filled pressure transducers attached to the forehead of test subjects allowing head loads to be assessed without kinematic or reverse dynamic analysis. However, the measured forces on the surface of the scalp may not represent the true forces experienced by the skull or brain. It has been documented that the scalp attenuates impact forces by up to 20 times $^{32}$ suggesting that accelerometers mounted to the scalp experience similar effects. Alternative systems employing intraoral accelerometers have been used to measure head responses but differed in response to the true skull measurements. ${ }^{28}$ For a series of helmeted cadaver head impacts, peak head accelerations measured from an intraoral device differed from measurements taken from the skull by $41-48 \%$, although excellent correlation between the two was found with a correlation coefficient $r$ of 0.84-0.94 for different impact conditions.

Electromyographic (EMG) studies complement kinetic and kinematic approaches by measuring the temporal characteristics of muscle activation, and hence external loading on the head. In a heading research study, the muscle activity levels and phasing were analysed in relation to heading approach (standing, jumping) and type (clearing, passing, and shooting). ${ }^{31}$ The levels of muscle activity were found to be inconsistent with the observations cited in an earlier study ${ }^{33}$ 
for different heading approaches and types. Other studies have employed EMG techniques to analyse the phasing of muscle activity of the sternocleidomastoid and trapezius muscles with heading biomechanics as these contribute greatly to head motion. ${ }^{31}{ }^{34}$ We therefore reviewed the implementation of surface EMG methods and used these as basis for the current study. ${ }^{31} 3536$

Analytical methods have been employed to gain insight into the biomechanical response of players under impact conditions. This type of methodology allows for the study of several parameters such as head mass and ball pressure without the variability commonly experienced with test subjects. In addition, internal forces available from the models can provide estimates of loads to the brain and cervical spine that would otherwise be difficult or impossible to assess with live subjects. However, the validity of the analytical predictions depends on the underlying assumptions of the model and accuracy of the human-ball interactions. Published studies employing numerical or analytical models have assessed the effects of heading, head mass variation, and ball mass/pressure characteristics. ${ }^{26}{ }^{37-39}$ This supplement includes a report of recent implementation of a detailed human model in conjunction with the current study to gain a better understanding of heading biomechanics. $^{40}$

\section{Biomechanical studies}

The implication of an association heading with cognitive dysfunction was assessed in a biomechanical study that included the analysis of two control groups. The first group consisted of players frequently exposed to heading (having more refined technique) and the second group consisting of players exposed infrequently to headings. ${ }^{58}$ Differences in heading techniques were suggested qualitatively to result in different head responses. ${ }^{172}$ In a separate attempt to quantify the effects of heading, a biomechanical study was undertaken on skilled subjects $(\mathrm{n}=4)$ and less skilled subjects $(n=6)$ to measure the effects of a single jumping heading technique on head acceleration response. ${ }^{23}$ The acceleration and deceleration phases of the head were used to infer head-neck-torso kinematics for comparison with an early pioneering study conducted by Mawdsley ${ }^{33}$ in 1978. Another biomechanical study investigated the kinematics of skilled and less skilled female players $(n=24) .{ }^{24}$ Neck and trunk motions between the two groups were quantified and related to heading technique. Head responses were not measured in this study.

The effects of heading type (shooting, clearing, and passing) and approach (jumping, standing) have been studied in relation to the forces exerted on the head and EMG neck muscle activity in collegiate female players $(n=15) .{ }^{31}$ The measured impact forces did not vary with header type or approach. The observations could not be further explained because of the lack of kinematic data.

\section{Analytical studies}

Analytical methods for gaining insight into heading biomechanics have been employed to study the effects of body mass and ball mass-pressure characteristics ${ }^{26}{ }^{37}$ Only the horizontal component of head acceleration was analysed with representations of the ball, head, and neck. The model was passive-that is, with the player standing still and not expecting the ball impact. It was found that ball mass, pressure, and flight characteristics influenced head response and varied with the effective mass (a function of player size, strength, and technique).

Additional analytical methods have been used in the study of football headgear wherein the brain response was estimated. The high level of detail in the passive head-neck model highlighted the ability to employ simulation techniques to analyse head kinematics as well as brain response. ${ }^{38}$ Passive heading motions were represented and the biofidelity was limited to the response of the neck model based on a car crash test dummy.

\section{Research objectives of the present study}

Current head injury research efforts deal largely with the diagnosis, treatment, and rehabilitation of the injuries. If injury reduction is to be managed through prevention or mitigation, a greater understanding of the aetiology and biomechanics is required. Biomechanical approaches provide a method to gain a better understanding of the underlying injury mechanisms and resulting injury risks. When combined with the incidence of injury, head impact conditions and clinical injury description, a powerful diagnostic tool can be developed. Both body kinematics and kinetics are needed to fully understand heading biomechanics. This requires direct measurement of head-neck-torso motions and neck muscle activity and phasing, as well as the direct measurement of head impact response. It is also apparent that due to large differences in subject heading techniques and body characteristics the experimental analysis must consider intersubject variations as well as whole population trends. Further, the use of analytical methods could potentially identify key parameters related to head response that would otherwise be problematic to assess with human subjects.

Thus the objectives of the current project were:

- to develop analysis techniques capable of measuring both kinematic and kinetic responses during heading

- to develop a numerical model for the study of biomechanical heading parameters

- to determine the effects of heading techniques on the measures of head impact response.

This paper is the first if a three part series in this supplement which deals with the development of biomechanical techniques for subject testing along with the results of the experiments. The development and results of the numerical modelling efforts form the subject matter for Part 2 "Biomechanics of ball heading and head response". ${ }^{40} \mathrm{~A}$ complementary study of ball impact response is presented in Part 3 "Effect of ball properties on head response". ${ }^{41}$ All three papers are based on the methodology described in this paper.

\section{METHODS}

Initial kinematic and kinetic responses were determined from volunteers performing a series of standard heading scenarios. The results provided a range of responses under typical heading manoeuvres for input into and validation of the numerical model. We then used the model for a detailed parametric analysis of heading biomechanics and generation of initial guidelines for the reduction of head impact severity. We confirmed and further investigated the guidelines with a smaller sample of test subjects. In this manner, we could either control or reduce poor repeatability associated with systematic and random errors resulting from experimental, subject, ball, and environmental effects. The ability to differentiate head response trends between heading techniques would be further improved through the use of intersubject analysis.

\section{Heading methods}

Many manuals, guides, and coaching techniques were reviewed to characterise the steps required to head the ball properly for the study. The three phases in heading include: pre-impact, ball contact, and follow through. The common methodology for frontal heading comprises the following: 
- Pre-impact

- feet placed in a split stance

- knees bent

- torso extended rearwards about the hips

- shoulders squared

- eyes fixed on the ball

- Ball contact

- torso flexed forwards to meet the ball

- head and shoulders move in unison with the torso

- head contacts ball at hairline

- Follow through

- torso and head motion continues immediately after contact and then decelerates to regain balance

The pre-impact phase allows the player to prepare to forcefully impact and direct the ball at the intended target. Ball contact with the forehead is recommended, not with the top of the head. Follow through is not widely discussed but is generally recommended to be in the direction of the target and in some cases, the head is thrust towards the target.

In general, headings can be categorised by their type and approach. The type describes the purpose for ball redirection: to clear the ball, pass it to another player, or to capture and gain control of the ball. These can be executed during different approaches, the commonest being standing, running, and jumping. The combination of heading type and approach can result in widely varying head responses. This variability is further increased when considering interpersonal and cross-player differences in heading technique, anthropometry, skill level, physiological state, existing injuries, and external factors such as ball speed and type, game situation (training, competition), and environment (wetness, temperature). For these reasons, we selected a limited number of heading and subject parameters for further investigation.

\section{Heading scenarios}

The heading approach and type selected for the human trials and the numerical simulations satisfied the following requirements:

- to achieve variations in head, neck, and torso alignment

- to explore neck muscle activity levels and phasing throughout the impact event

- to investigate different heading types for different ball speeds.

The requirements were achieved through the selection of various ball placement targets that allowed the natural selection of muscle activation and technique by each player, through the use of different ball approach speeds.
The selected heading scenarios, detailed in table 1 , consist of three ball placement targets, and two ball speeds for frontal impact with the forehead. Three neck muscle activity levels (normal, pre-tensed, relaxed) and four body motions (normal, follow through, aligned, and passive) further complement the test matrix for a total of 10 heading scenarios. Four additional scenarios were introduced to address ball mass and pressure variations for a single heading configuration and are reported on separately. ${ }^{41}$

We used a subset of the heading scenarios for the initial subject trials $(\mathrm{n}=7)$ to determine the overall response envelope and for implementing and validating the numerical model. These are designated as heading codes LS1, LS2, LS3, and HS3. We selected the LS2 condition as the reference or baseline condition for future comparisons. Additional tests established the range of repeatability for a given subject for each of the impact severity measures. A series of three repeated impacts were conducted with the baseline heading configuration (LS2) for two subjects (HS6, HS12). The remainder of the heading scenarios were based on a single test once acceptable data were acquired.

The results from the initial subject tests and numerical simulations guided a second test series with fewer human subjects $(n=3)$. The purpose of this series was to verify the kinematic and kinetic responses with more detailed measurements and to conduct a parameter study of heading techniques that would lead to a better understanding of the biomechanics. We employed the complete matrix in table 1 for these trials.

The pre-tensed neck muscle condition attempts to couple the head better with the neck and torso. This was incorporated in the low and high speed ball placement scenarios (LS2-MT, HS3-MT). The test subjects were instructed to provide maximal voluntary tensing while keeping the head in a similar attitude as for the normal condition (LS2). A balance of the flexor and extensor muscle groups is required and generally involves upper body tensing. A relaxed condition (HS3-RLX) was used to emulate an unprepared player being struck by the ball on the forehead. This poor level of head coupling with the body was studied to investigate changes in head response with minimal neck muscle activity. The subjects were instructed to remain standing and not to contribute actively to the impact. Player follow through (LS2-FT) was introduced to increase the duration of muscle activation for the sternocleidomastoid and trapezius muscles through the impact event. Alignment between the torso, neck, and head (LS2-TA) was investigated to study the effects of head coupling with the skeletal system. The alignment process attempted to align:

- the ball impact with the centre of gravity of the head to help reduce head rotations, and hence uncoupling of the head

\begin{tabular}{lllll}
\multicolumn{4}{l}{ Table 1} & Heading scenarios for human subjects \\
\hline \multicolumn{2}{l}{ Heading scenario } & Ball speed Modification & Ball target & Heading code \\
\hline Controlling & Low & None & Front, down, $2.75 \mathrm{~m}$ from player & LS1 \\
Passing & Low & None & Front, down, $5.5 \mathrm{~m}$ from player & LS2 \\
Clearing & Low & None & Up and away, as far as possible & LS3 \\
Clearing & High & None & Up and away, as far as possible & HS3 \\
Clearing & High & Neck muscle tensing & Up and away, as far as possible & HS3-MT \\
Head rebound & High & Neck muscles relaxed & None & HS3-RLX \\
Passing & Low & Neck muscle tensing & Front, down, $5.5 \mathrm{~m}$ from player & LS2-MT \\
Passing & Low & Follow through & Front, down, $5.5 \mathrm{~m}$ from player & LS2-FT \\
Passing & Low & Torso alignment & Front, down, $5.5 \mathrm{~m}$ from player & LS2-TA \\
Passing & NA & No ball impact & Front, down, $5.5 \mathrm{~m}$ from player & LS2-NI \\
\hline
\end{tabular}


- the ball impact trajectory with the longitudinal axis of the cervical spine providing good neck support

- the cervical and thoracic spines to provide a rigid support for the head.

The test subjects were instructed to place their torso in a more horizontal orientation, roughly in the line of the ball trajectory. Ball contact occured with the head in a more extended position providing the desired alignment. The new orientation may not be suitable for jumping approaches but serves to provide a better understanding of the load transmission and body coupling. In the final configuration the subjects headed the ball without actual ball contact. This was meant to provide a reference for understanding the active response of the neck muscles to ball impact.

\section{Ball speed}

Selection of the ball speed during heading will greatly affect the overall response of the head and may mask the effects of changes to heading technique. The kicked speed of balls varies considerably depending on the size, strength, and skill level of the player. Peak values in the range of $17.8 \mathrm{~m} / \mathrm{s}$ have been cited as being typical in a high school game, ${ }^{25}$ and higher peak values of $25 \mathrm{~m} / \mathrm{s}$ and $17-33 \mathrm{~m} / \mathrm{s}$ have been noted for professional players. ${ }^{1134}$ The speed at which balls are purposefully headed are undoubtedly lower and vary with player age, skill level, and game type. A numerical analysis of ball speeds based on the time and flight distance data from competitions indicated that the horizontal ball velocities are similar between youths and adults despite the strength and size differences of the players. ${ }^{37}$ A horizontal velocity range of $1-14 \mathrm{~m} / \mathrm{s}$ was noted with a mean speed of $5.7 \mathrm{~m} / \mathrm{s}$ for adults and $7.1 \mathrm{~m} / \mathrm{s}$ for youths. The mean speed for youths was higher than for adults despite the lower ball departure speeds from the foot. Previous research studies have employed heading ball speeds of $6.3-12 \mathrm{~m} / \mathrm{s}^{24} 2531$

We selected nominal ball speeds of $6 \mathrm{~m} / \mathrm{s}$ and $8 \mathrm{~m} / \mathrm{s}$ for the low speed (LS) and high speed (HS) intentional heading scenarios, respectively. We felt these adequately represented the range of ball heading speeds while limiting the exposure of the test subjects. Note that the closing speed between the moving head and ball is higher due to the contribution of forward head motion. Forward head speeds of $1-4 \mathrm{~m} / \mathrm{s}$ were noted for the current study resulting in head to ball closing speeds up to $11 \mathrm{~m} / \mathrm{s}$.

\section{Subject selection, screening, and welfare}

Seven test subjects, aged 20-23 years, took part in the initial study and a subset of three was chosen for the final verification. All subjects were active participants in nonprofessional football with 5-13 years' experience. All subjects underwent medical examination, and the Ottawa Hospital Research Ethics Board approved the protocols. A licensed physician was in attendance for all testing.

Anthropometric screening was conducted to approximate the typical adult male player and to ensure similitude with the numerical model (50th percentile male). We obtained stature and weight criteria from a worldwide anthropometric survey, ${ }^{42}$ and these are presented in table 2 along with the subject data.

Anatomical and video targets were applied to the subjects (fig 1), with their anatomical location measured with an electromechanical three dimensional digitiser (Bronze series FARO arm; FARO Technologies Inc., Lake Mary, FL) prior to testing. The head anatomical coordinate system was defined by the Frankfurt plane (tragion-orbitale) and the intraoral instrumentation bracket (targets $\mathrm{Al}-\mathrm{A} 2$ ). Torso alignment was measured with a rigid bar mounted to the surface of the superior and inferior aspects of the thoracic spine (targets
S1-S2). A contoured stiff foam pad under the bar maintained alignment with the torso.

\section{Instrumentation}

We used high speed video and reflective head and torso targets for kinematic measurement of the test subjects. Video data were captured with a Motion Scope Model 1000 high speed video camera (Redlake, USA) set for a capture rate of 500 frames per second (fps). A telephoto lens ( $6 \mathrm{~mm}, \mathrm{fl}: 1.2$ ) and large subject to camera distance were used to minimise parallax errors within the field of view. The video images were captured in digital format and the target positions were quantified in the midsagittal plane with frame digitisation and automated two dimensional tracking software for the verification test series.

We used two different types of intraoral device, capable of measuring linear and angular accelerations, to measure the head kinetics. The units were custom fitted to individuals with thermoformed bite plates to ensure good coupling with the skull. The unit seen in fig 1 was used for the initial human trials and allowed for three dimensional measurement but was prone to vibrations due to the cantilevered mass at the mouth. A refined unit was used for the verification trials and provided linear and angular accelerations in the midsagittal plane (fig 2). Subjects clenched the bite plate firmly in both cases.

We measured linear accelerations with Endevco Model 7264-2000 piezo-resistive accelerometers and angular acceleration with an Endevco Model 7302BM4 angular accelerometer (Endevco Corp., San Juan Capistrano, CA). This method of direct angular acceleration measurement provided repeatable estimates in comparison to the linear accelerometer array used in the first device. All kinetic measurements were taken at the origin of the device with its location digitised. The available bandwidth of the measurement system was better than $1000 \mathrm{~Hz}$.

Neck muscle activity was measured with EMG techniques. Due to the low level of electrical voltage, the technique is often plagued with artefacts from local noise sources. To address this, a bipolar electrode system, developed by $\mathrm{C}$ DeLuca and available through Delsys Inc., (Boston, MA) was selected due to its inherent high signal to noise ratio. The system uses two electrodes for each measurement site along with amplification at the electrodes and an additive differential amplifier. Other factors contributing to EMG measurement errors were the movement of the surface mounted electrodes over the muscle bundles or the movement of muscle groups under the electrodes. Surface mounted electrodes were preferable over invasive needle electrode EMG measurement techniques due to the anticipated movement of the neck muscles during heading. The location of the electrodes was based on the accessible muscle groups responsible for neck flexion and extension. The major muscle groups responsible for movement of the head are the sternocleidomastoid, the semispinalis capitis, the splenius capitis, the longissimus capitis, and the trapezius. ${ }^{43}$ The left and right sternocleidomastoid and trapezius were therefore

\begin{tabular}{lll}
$\begin{array}{l}\text { Table } 2 \\
\text { data }\end{array}$ & Anthropometric and range of motion \\
\hline Description & Requirements & $\begin{array}{l}\text { Subjects } \\
\text { (mean (SD)) }\end{array}$ \\
\hline Stature $(\mathrm{cm})$ & $175.3(2.6)$ & $171.5(7.9)$ \\
Weight $(\mathrm{kg})$ & $77.3(3.4)$ & $73(11)$ \\
Head circumference $(\mathrm{cm})$ & $57.1(1.9)$ & $56.9(2.0)$ \\
Neck circumference $(\mathrm{cm})$ & $38.3(1.5)$ & $38.1(3.0)$ \\
\hline
\end{tabular}




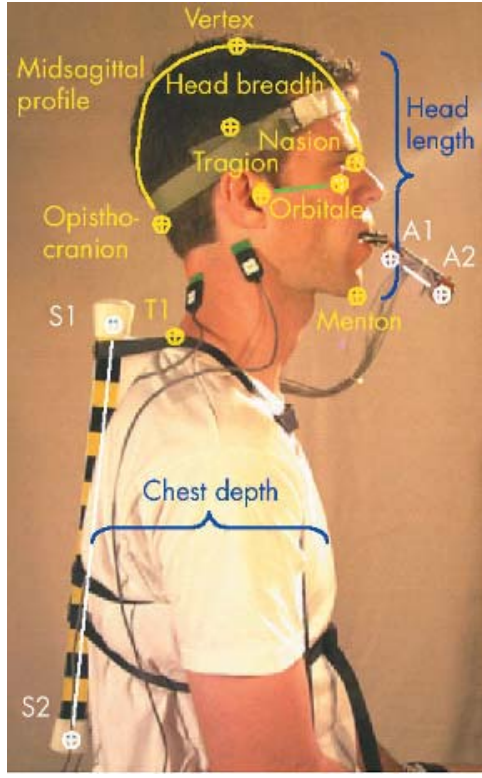

Figure 1 Reference marks and data.

selected as these are the most superficial and have been used in previous studies. ${ }^{31}$ We placed an additional electrode over the back of the elbow (olecranon process of the ulna) to provide an electrical reference to the circuit.

Characterisation of EMG responses can be problematic as averaged results of several subjects do not result in meaningful data due to intersubject variations with muscle activity level and phasing (Dr Krabbe, McGill University, personal communication, 2002). Our preferred approach in this study was to select a single subject exhibiting average EMG activity and compare the responses within the subject.

\section{Data collection and conditioning}

We used a data acquisition system at 10000 samples per second (National Instruments Model PCI-6110; National Instruments Inc., Austin, TX) to record the accelerometer and EMG electrode data. Data collection was initiated with a thin foil contact switch located on the subject's forehead. A data pre-sampling window of $200 \mathrm{~ms}$ allowed the pre-impact kinetics of the subject to be measured while a recording duration of $200 \mathrm{~ms}$ after impact was sufficient to capture the follow through dynamics.

The linear accelerometers were conditioned with a differential amplifier (Endevco Model 136) and passive antialiasing low-pass filter of $1000 \mathrm{~Hz}$ (meeting the requirements of the Society of Automotive Engineers' Guideline J211-1, CFC 1000). Post-test low-pass filtering of the linear and angular accelerometers to $180 \mathrm{~Hz}$ was carried out based on previously established practices. $^{44}$ The EMG data were amplified and conditioned with a built-in passive antialiasing low-pass filter of $500 \mathrm{~Hz}$.

The EMG data were post processed to compute the root mean square (RMS) value of the rectified signal and normalised with the value corresponding to $100 \%$ mean voluntary contraction (MVC). Typical amplitudes of EMG signals can range from $0 \mathrm{mV}$ to $10 \mathrm{mV}$ (peak to peak) or from $0 \mathrm{mV}$ to $1.5 \mathrm{mV}$ (RMS). The usable energy of the signal is contained within the $0-500 \mathrm{~Hz}$ frequency range with the dominant energy being in the $50-150 \mathrm{~Hz}$ range. A custom subtractive filtering algorithm was developed and implemented in Matlab to remove the mains noise $(60 \mathrm{~Hz})$ without altering the true magnitude of the muscle activity. This was applied prior to computing the rectified and RMS EMG data.

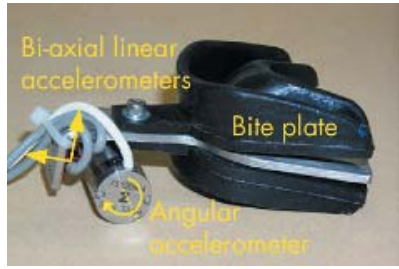

Figure 2 Intraoral device.

Normalisation was not completed due to higher muscle activity levels recorded during the dynamics of heading compared with the MVC. This is thought to be attributed to differences in the muscle line of action, number of muscles being excited, and submaximal excitation during static measurement. Although an absolute measure of muscle force was not attainable, this was of no consequence for the intersubject comparisons.

\section{Head impact severity assessment}

Skull, brain, brainstem, and spinal injuries are known to result from some form of mechanical insult to the head, either through direct loading (that is, impact) or through indirect (that is, inertial) loading. These can result in global motions of the head (translational/rotational) and local deformations leading to physiological and mechanical injuries. Early research efforts suggested that compressive (pressure) and shear stresses are the mechanisms responsible for injury but their direct relation to tissue disruption and physiological dysfunction have been the subject of much research. Although direct measure of tissue response and correlation with injury is desirable, it is difficult to achieve and requires human or subhuman primate test subjects. Alternative approaches with numerical models to predict tissue responses are also under way but again require validation from in situ or in vivo experiments.

Practical approaches to developing biomechanical injury criteria have been based on the correlation of mechanical insult with the injury outcome. Early research efforts demonstrated decreased tolerance of the brain with increased duration of loading and magnitude of translational/rotational accelerations of the head. This time dependency was characterised in the Wayne State Tolerance Curve (WSTC) which presents the occurrence of concussion as a function of linear acceleration and time duration. ${ }^{45}$ This was initially based on the response of embalmed cadavers under rigid head impacts resulting in linear skull fractures with loading durations of $1-6 \mathrm{~ms}$. The occurrence of skull fracture was proposed to indicate the onset of cerebral concussion despite the fact that most concussions do not involve fractures. The durations were later extended up to $100 \mathrm{~ms}$ by incorporating animal, cadaver, and human volunteer tests. This was later reformulated into the weighted impulse criteria for assessing acute brain injuries. The Gadd Severity Index and the head injury criterion are examples of such criteria currently referenced in standards for helmet performance and head injuries in automobile impacts, respectively. The WSTC was later confirmed and updated in the Japan Head Tolerance Curve and was the basis for a motorcycle helmet standard (FMVSS 218) where tolerable linear acceleration limits are specified for different durations.

The contribution of rotational loads to brain injury have been addressed through research studies focused on rotationally induced mechanisms. ${ }^{46-48}$ Brain injury tolerance limits derived from primate studies were proposed with severity increasing with increasing angular acceleration magnitude and angular velocity change. The relations between magnitude and velocity change with injury outcome exhibit significant discrepancies among the various studies. 
Efforts to include both linear and rotational criteria have been made with the combination of normalised linear and angular accelerations, the Generalized Acceleration Model for Brain Injury Tolerance (GAMBIT). ${ }^{49}{ }^{50}$ The model has been validated under limited conditions but has been criticised on the grounds that it does not account for the time dependency of head injury tolerance.

Validation of the various injury criteria have been primarily focused on direct or inertial loading conditions producing acute injury or traumatic brain injury (TBI). The suitability of these for addressing injuries of lower severity, - that is, concussion-is not known due to the limited availability of data describing the relation between severity of mechanical insult and injury outcome. Further, the suitability to repetitive sub-concussive impacts is not known and is compounded by the difficulty in assessing the level of neurophysiological and neuropsychological dysfunction.

Recent work sponsored by the American National Football League (NFL) investigated the relation between linear and rotational head kinematics and the occurrence of mild traumatic brain injury (MTBI). Through the reconstruction of game impacts, a Head Impact Power (HIP) index was formulated and validated with documented cases involving concussion and the absence of concussion..$^{51}$ This was found to have a better predictive power of MTBI than criteria associated with TBI.

The HIP criterion is based on the rate of energy transfer to the head and employs both linear and angular accelerations for all degrees of freedom. With the use of mass and inertial characteristics for a 50th percentile male head, represented by a Hybrid III automotive test dummy (Denton ATD Inc., Milan, OH) in the current study, the HIP is defined according to the following formula:

$$
\begin{aligned}
H I P= & 4.50 a_{x} \int a_{x} d t+4.50 a_{y} \int a_{y} d t+4.50 a_{z} \int a_{z} d t+ \\
& 0.016 \alpha_{x} \int \alpha_{x} d t+0.024 \alpha_{y} \int \alpha_{y} d t+0.022 \alpha_{z} \int \alpha_{z} d t
\end{aligned}
$$

where

$a_{i}=$ linear acceleration at the head's centre of gravity about anatomical coordinate axis $i(i=x, y, z),\left(\mathrm{m} / \mathrm{s}^{2}\right)$

$\alpha_{i}=$ rotational acceleration about axis $i,\left(\mathrm{rad} / \mathrm{s}^{2}\right)$.

To provide a clear indication of ball impact severity, the power index was computed starting at the time of ball-head contact rather than starting at the beginning of body motion as conducted in the NFL study. ${ }^{51}$ Note that the portion of power attributed to head movement required to meet the ball represents a large component of the overall peak power seen by the head. By ignoring the pre-impact kinematics, only the incremental power increase from the ball is represented.

The probability of MTBI for American football is presented in the form of a logistic regression curve where the probability of injury can be assessed from a measured head response. Validity of the criteria is limited to the conditions of the re-enactments.

$$
P=\frac{1}{1+e^{-(\alpha+\beta X)}}
$$

where

$$
\alpha=-4.605, \beta=0.356, X=H I P
$$

Repetitive low severity impacts have been speculated to contribute to neurophysiological and neuropsychological dysfunction of the brain or CTBI. No studies have formulated a criterion relating the degree of mechanical insult to the occurrence of CTBI. In the absence of such criterion and the

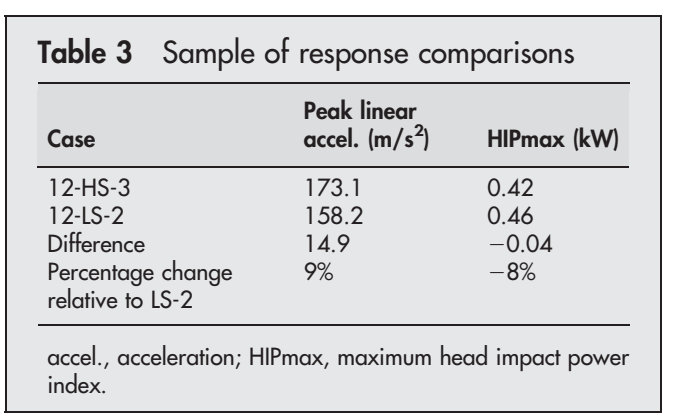

limitations of those associated with TBI, we employed HIP as a measure of impact severity, not injury, based on the hypothesis that a criterion which is validated under conditions most similar to those in the current study will be most appropriate overall. Peak linear and angular accelerations were also used for comparisons with earlier studies.

\section{Intersubject comparisons}

At the onset of the study, it was recognised that a biomechanical comparison of the human subjects would have inconsistencies due to unique variations within each subject with regard to heading technique, anthropometry, skill level, and strength. The intersubject comparisons were based on the relative change in response to a baseline test. The low speed heading scenario (LS2) was used as the baseline with the change in response for a different scenario-for example, HS3 - expressed as a percentage of the baseline magnitude. In this case, a relative increase in peak linear acceleration of $9 \%$ was observed as detailed in table 3. A similar comparison with the HIP index showed an $8 \%$ decrease relative to the low speed heading configuration. Similar comparisons were conducted for all injury metrics and an increase in response is indicated by a positive number and a decrease as negative.

\section{RESULTS \\ Initial subject trials \\ Kinematic data}

Kinematic data for the initial trials were manually digitised limiting the accuracy to the resolution of the video and approximation of the targets by the operator. Kinematic plots of the individual subjects are presented in fig 3 depicting the differences in initial posture and follow through of the body segments during heading.

The torso angle depicted in fig 3B presents the inclination of the torso with respect to the laboratory reference frame. Vertical orientation is at $0^{\circ}$ and a rearwards orientation is represented by negative values. The time base corresponds to the actual event where ball impact occurs at 0 ms. It can be observed from the linear slope of these curves that the rate of torso rotation of each subject was relatively constant during this time period with the only difference being the initial inclination angle. The change in torso angle also indicated an active involvement of the torso contributing to the overall heading performance. Human subject HSil demonstrated rudimentary heading skills and was removed from the analysis.

The kinematic data for the head (fig 3A) depict the orientation of the Frankfurt plane relative to the laboratory reference frame with $0^{\circ}$ being horizontal and positive corresponding to the chin down attitude. Differences between the initial head angles were observed along with minor differences in the rate of head rotation. Note that the head rotations in themselves do not fully correspond to heading performance as the rotation of the torso contributes to the observed head angles. 
A

LS2-Head angle

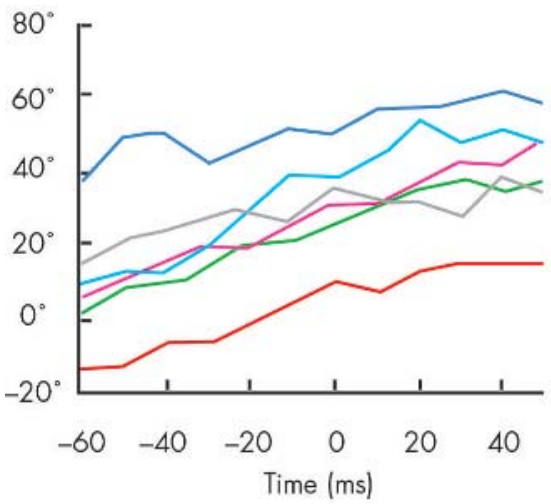

C LS2-Relative head-back angle

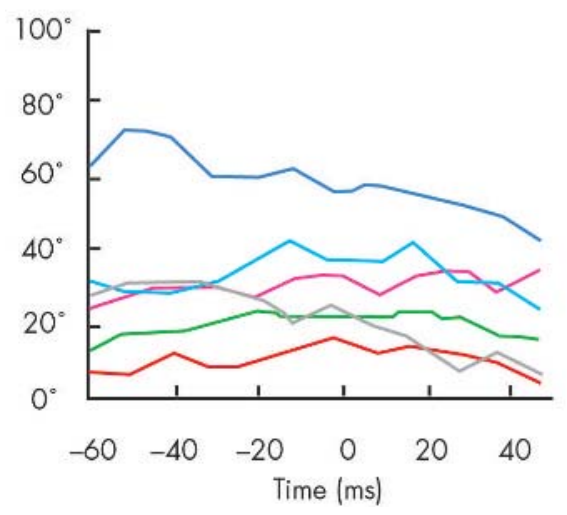

B LS2-Back angle

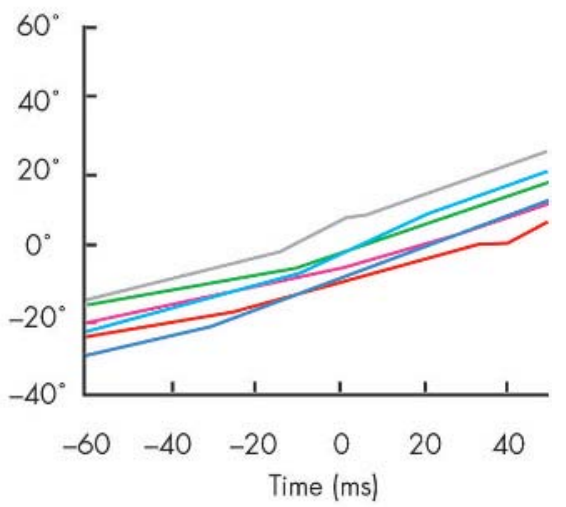

D LS2-Change in relative head-

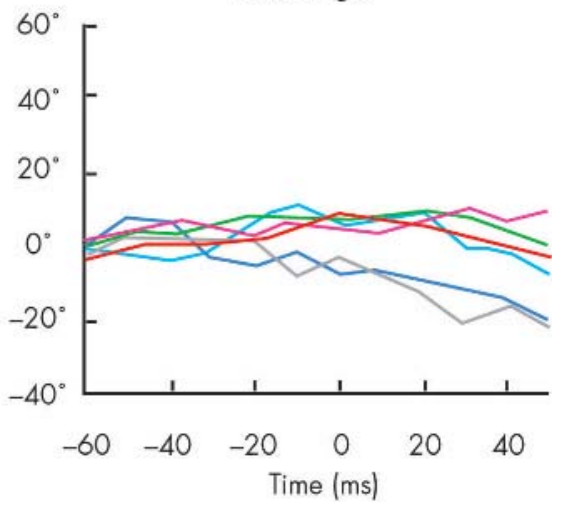

Figure 3A-D Subject kinematics for the baseline heading scenario (LS2).
Differences in the head angle will affect the contact point with the head, the interaction of the neck muscles, and the resulting head response. Figure $3 \mathrm{C}$ depicts the relative head to torso angles where a positive angle corresponds to head flexion. Again, the relative angles are different across subjects with the majority keeping their head locked relative to the torso. The range of head rotation relative to the torso is depicted in fig 3D where the responses are forced to start at the same angle at $-60 \mathrm{~ms}$. Some subjects were noted to flex their necks (chin to chest) whereas others extended their necks (head back).

Similar observations were noted for all heading scenarios with the greatest variation for the high speed clearing shot (HS3) (fig 4). Less torso rotation was observed along with a greater head to torso angle resulting in a more horizontal attitude suitable for directing the ball upwards and forwards.

\section{Kinetic results}

The resultant acceleration of the head during ball impact was based on the instrumentation located at the mouth. The resultant linear acceleration indicated a wide range of peak head responses across the subjects. These are likely a result of differences in head impact velocity, neck muscle activity, body orientations, and ball rebound velocity. Although the magnitudes varied between the subjects, the time to peak and characteristics were remarkably similar (fig 5). Accelerations are expressed as gravitational units $\left(1 \mathrm{~g}=9.81 \mathrm{~m} / \mathrm{s}^{2}\right)$. The dual peaks are due to resultant accelerations always being positive after vector addition of the fore-aft and inferior-superior components. A sinusoidal response of the components is typically observed due to head rotations during impact.

\section{Neck muscle activity}

The muscle activation timing for the heading scenarios are presented in fig 6 for all subjects except one (HS15). The durations are based on the approximate time at which the activity levels, defined by the RMS of the EMG (EMG $\mathrm{RMS}_{\text {), }}$ begin to rise and fall. The peak activation level typically occurs midway.

The data demonstrate that the left and right sternocleidomastoid muscle groups are activated $280-500$ ms prior to ball impact for all subjects with the peak effort occurring between 90-150 ms before impact. For the heading scenarios involving precise ball placement (LS1, LS2) the sternocleidomastoid muscles became inactive at the time of, or just after, impact. The trapezius muscles are also activated prior to impact and remain so after ball departure to stabilise the head during follow through. Some asymmetry exists between the left and right sides and is attributed to the open stance employed by the subjects with the torso oblique and the neck twisted to face the ball head-on. The low and high speed ball clearing heading scenarios (LS3, HS3) required greater effort from the muscles and therefore resulted in longer activation times and greater follow through.

The timing and relative contributions of the sternocleidomastoid and trapezius muscle groups are of greatest interest in the current study as these reflect the heading techniques used by the subjects.

\section{Head response repeatability}

The coefficient of variation $(\mathrm{CV})$ for the peak linear accelerations was in the range of $5-12 \%$ and can be attributed to differences in the ball impact velocity, head kinematics at impact, vibrations of the intraoral device, and neck muscle 


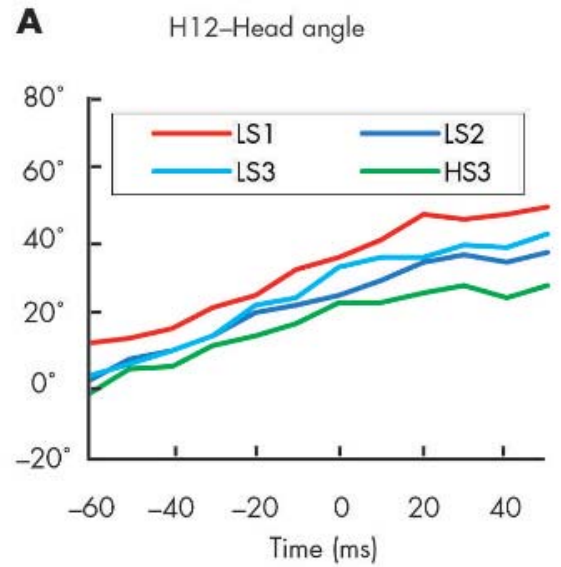

C HS12-Relative head-back angle

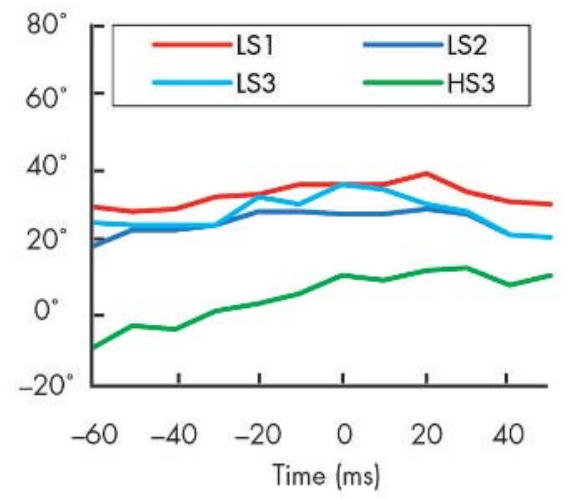

B

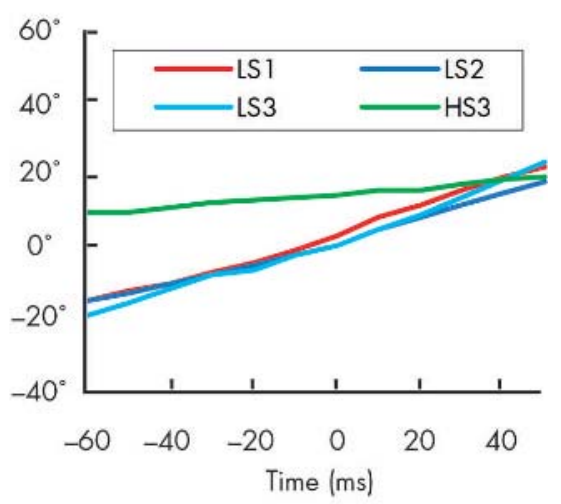

D HS12-Change in relative headback angle

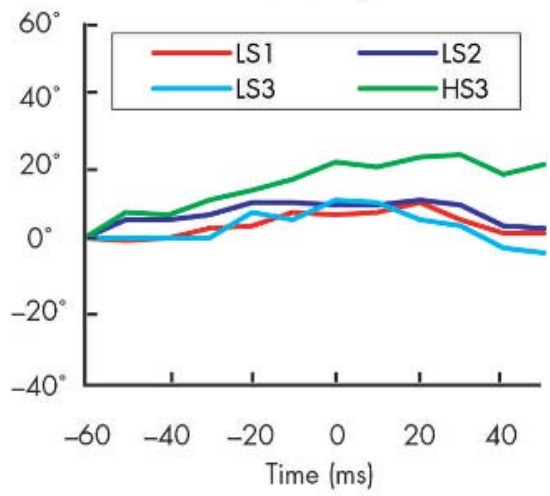

Figure 4A-D Single subject kinematics for various heading scenarios. See table 1 for explanation of heading scenario codes. activity. These factors are treated as random errors and contribute to the overall variability. The systematic errors introduced by the instrumentation, data acquisition process, and scaling errors are estimated to be less than 5\% and contribute to the observed CV.

The peak angular accelerations exhibited a CV of $5-19 \%$. This is due partially to variations in the impact conditions as mentioned previously and to the semirigid coupling of the intraoral device to the skull. The HIP exhibited higher CVs of $37-41 \%$. This is not unexpected, as the power index is the product of the accelerations and velocities for the linear and angular components.

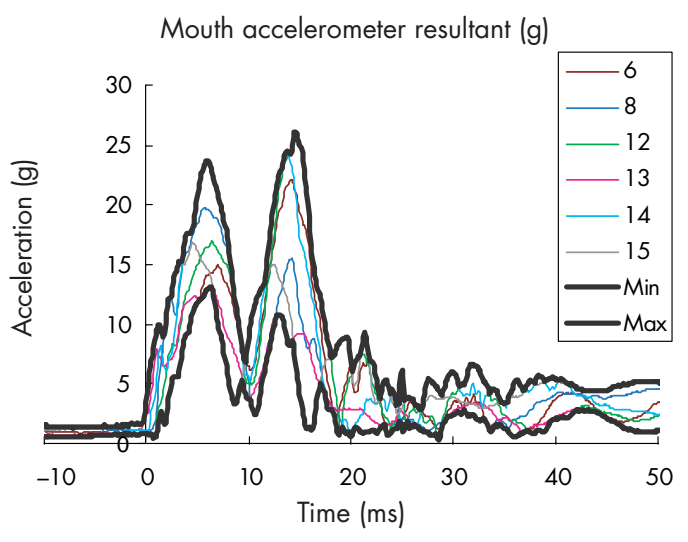

Figure 5 Subject resultant linear accelerations.

\section{Subject verifications}

Kinematic data

Analysis of the kinematic data for the expanded heading scenario test series differed from the initial subject kinematics in that the focus was on the initial dynamic conditions of the head just prior to ball contact. The initial velocity and

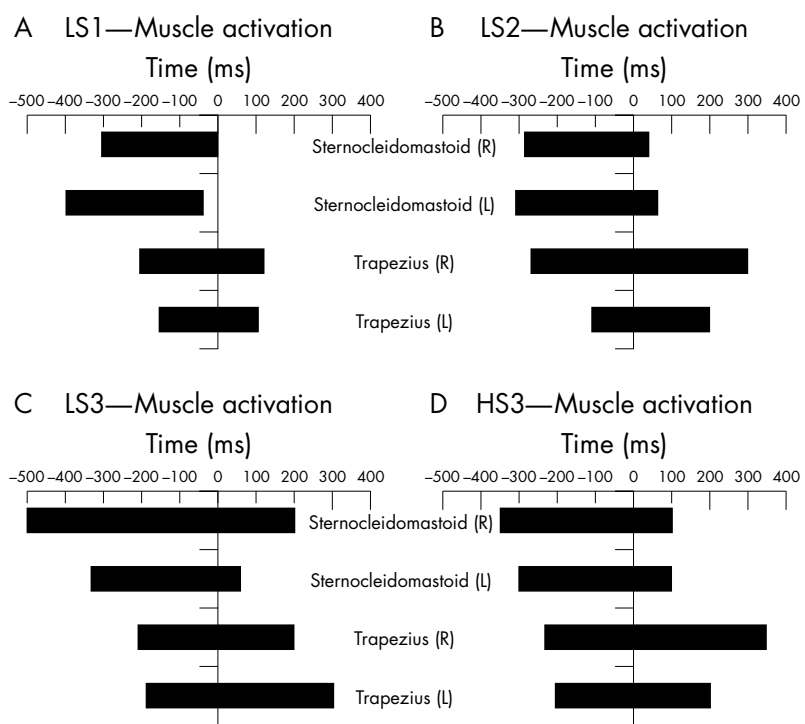

Figure 6A-D Neck muscle activation times for all heading scenarios. See table 1 for explanation of heading codes. 
orientation of the head and ball predominantly describe differences in the impact severity and subsequent conclusions are drawn from comparisons.

Averaged head and ball kinematics across all subjects are given in table 4 . The average head angle was inclined downwards $\left(-4^{\circ}\right.$ to $\left.-33^{\circ}\right)$ for all heading scenarios with ball contact. A greater downward angle was noted for heading types involving redirection of the ball whereas a more horizontal attitude was seen for the clearing scenarios. A slight upwards orientation was noted for the non-impact scenario (LS2-NI) and is likely due to the difficulty in judging the relative position of the head and body to an imaginary ball. Average head velocities varied from $2.5 \mathrm{~m} / \mathrm{s}$ to $3.2 \mathrm{~m} / \mathrm{s}$ for cases with ball contact resulting in ball-head closing speeds of $7.5-10.3 \mathrm{~m} / \mathrm{s}$. Higher head speeds occurred in clearing heading scenarios. Clearing scenarios typically resulted in shallower ball-head angles $\left(8^{\circ}-45^{\circ}\right)$ whereas those involving greater redirection had greater angles $\left(58^{\circ}-\right.$ $76^{\circ}$ ).

\section{Kinetic data}

The head responses exhibited similar characteristics to the initial subject trials. Linear and angular accelerations responses for HS12 are shown in fig 7. The mean and standard deviation bounds are provided and are based on the repeatability tests described earlier.

Head responses for all heading scenarios are summarised in table 5 for the human subjects where " $\mathrm{n}$ " represents the number of subjects. These are expressed as resultant linear and angular accelerations with the lowest and highest peak values. The accelerations were measured at the location of the intraoral device providing linear and angular responses in the midsagittal plane. For heading scenarios involving ball contact, the average peak accelerations varied from $14 \mathrm{~g}$ to $20 \mathrm{~g}$ with impact durations ranging from $14 \mathrm{~ms}$ to $33 \mathrm{~ms}$. Similar results were observed for the angular accelerations which exhibit an average peak range of 1.4-2.41 krad $/ \mathrm{s}^{2}$.

The HIP values are based on the sum of the linear and angular components calculated from the time of impact. Average peak impact power values of 246-562 W were measured for heading scenarios involving ball contact. These are presented at the location of the intraoral device.

A composite plot of the average data is presented in fig 8 which depicts the peak linear accelerations and maximum HIP values. The values are substantially below the risks associated with $\mathrm{MTBI}^{51}$ suggesting that the differences between the accelerations at the centre of gravity of the mouth and head are not pertinent.

\section{Neck muscle activity}

The rectified $E M G_{R M S}$ responses for the heading scenarios exhibited phasing trends similar to the initial subject trials.

\begin{tabular}{|c|c|c|c|c|c|c|}
\hline $\begin{array}{l}\text { Heading } \\
\text { scenario }\end{array}$ & $\begin{array}{l}\text { Head } \\
\text { angle } \\
\text { (deg) }\end{array}$ & $\begin{array}{l}\text { Head } \\
\text { velocity } \\
\text { angle } \\
\text { (deg) }\end{array}$ & $\begin{array}{l}\text { Ball } \\
\text { velocity } \\
\text { angle } \\
\text { (deg) }\end{array}$ & $\begin{array}{l}\text { Head/ball } \\
\text { velocity } \\
\text { angle } \\
\text { (deg) }\end{array}$ & $\begin{array}{l}\text { Head } \\
\text { velocity } \\
(\mathrm{m} / \mathrm{s})\end{array}$ & $\begin{array}{l}\text { Head/ball } \\
\text { velocity } \\
(\mathrm{m} / \mathrm{s})\end{array}$ \\
\hline HS3 & -4 & 24 & 32 & -8 & 2.5 & 10.3 \\
\hline HS3-MT & -16 & -1 & 42 & -43 & 3.1 & 9.8 \\
\hline HS3-RLX & -5 & -29 & 33 & -62 & 0.2 & 5.3 \\
\hline LS1 & -33 & -19 & 57 & -76 & 2.5 & 7.5 \\
\hline LS2 & -18 & -7 & 58 & -65 & 3.2 & 8.0 \\
\hline LS2-FT & -14 & -1 & 56 & -58 & 2.8 & 8.0 \\
\hline LS2-MT & -18 & -9 & 55 & -64 & 3.0 & 7.9 \\
\hline LS2-NI & 10 & 13 & NA & NA & 0.9 & 0.9 \\
\hline LS2-TA & -15 & -16 & 57 & -72 & 2.8 & 7.5 \\
\hline LS3 & -9 & 10 & 55 & -45 & 3.2 & 9.0 \\
\hline
\end{tabular}
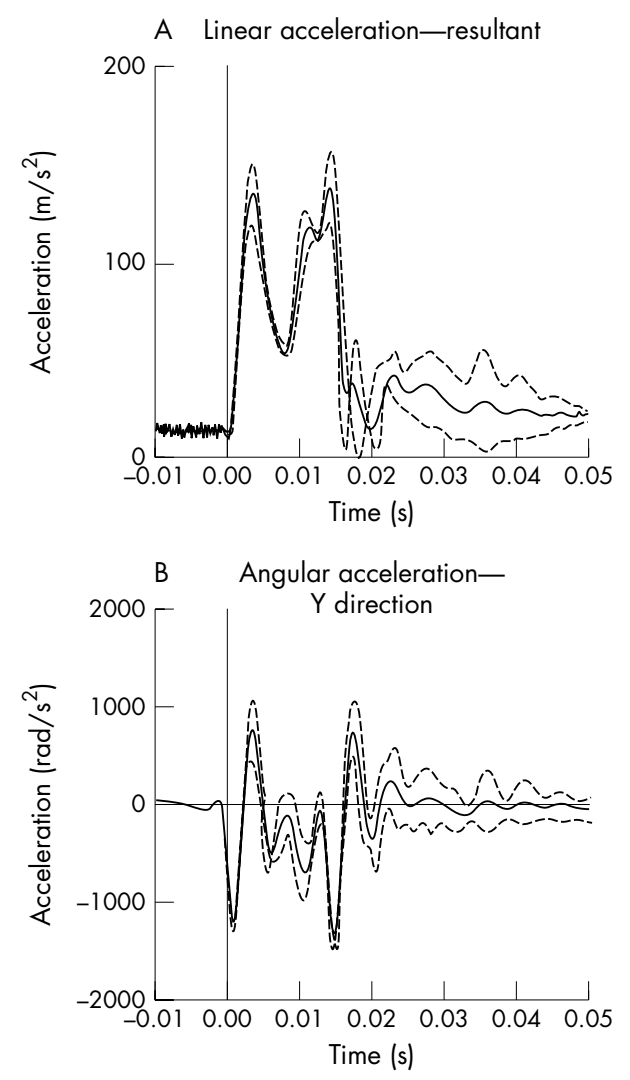

Figure 7A,B Head linear and angular accelerations (mean (SD)).

The sternocleidomastoids were active prior to and following the impact event up to $60 \mathrm{~ms}$ after impact. In the former cases, activity is attributed to stabilisation of the head during movement of the body to meet the ball and to forcing the head forward for greater ball impact. Activation of the trapezius muscle groups prior to impact was observed but at a much lower level of effort as these act to stabilise the head and contribute minimally to the heading. For the pre-tensed scenarios, muscle activity was elevated.

The integrated muscle response indicated the level of contribution to the head motions. This proved useful when comparing the various heading scenarios. It can be seen in fig 9 that the sternocleidomastoid activity diminished greatly after ball contact (0 seconds) with similar reduction in the integrated response. Figure 9 depicts the mean response and standard deviation bounds for subject HSI2 in the baseline scenario.

\section{Ball input/rebound speeds}

The incoming speed of the ball was nominally $6 \mathrm{~m} / \mathrm{s}$ and $8 \mathrm{~m} / \mathrm{s}$ for the low and high speed heading scenarios, respectively. The average speeds achieved for the verifications tests were: 6.2 (SD 0.3$) \mathrm{m} / \mathrm{s}$ for the low speed headings and 7.6 (SD 0.5$) \mathrm{m} / \mathrm{s}$ for the high speed tests. When considering the head approach velocity and ball incoming velocity, the average closing speed was increased to 7.6 (SD 1.3) m/s for the low speed headings and 10.1 (SD 1.1) m/s for the high speed tests.

In heading scenarios with aimed ball redirection, the ball rebound speed was greater than the incoming speed indicating that energy was being added to the ball by the subject. On average, the kinetic energy of the ball was increased by $85 \%$ to $137 \%$ with the clearing shots having the greatest increase. 
Table 5 Summary of human subject data

\begin{tabular}{|c|c|c|c|c|c|c|c|c|c|c|c|c|c|}
\hline \multirow[b]{2}{*}{ No } & \multirow[b]{2}{*}{ Scenario } & \multicolumn{4}{|c|}{ Peak linear acceleration $\left(\mathrm{m} / \mathrm{s}^{2}\right)$} & \multicolumn{4}{|c|}{ Peak angular acceleration $\left(\mathrm{krad} / \mathrm{s}^{2}\right)$} & \multicolumn{4}{|c|}{ Peak impact power (W) } \\
\hline & & Min. & Max. & Avg. & SD & Min. & Max. & Avg. & SD & Min. & Max. & Avg. & SD \\
\hline 3 & HS3 & 141 & 173 & 158 & 16 & 1.05 & 1.74 & 1.46 & 0.36 & 329 & 444 & 399 & 61 \\
\hline 3 & HS3-MT & 159 & 193 & 171 & 20 & 1.34 & 1.76 & 1.54 & 0.21 & 225 & 554 & 420 & 173 \\
\hline 2 & HS3-RLX & 169 & 180 & 175 & 8 & 1.52 & 1.77 & 1.64 & 0.17 & 368 & 415 & 391 & 33 \\
\hline 3 & LSI & 151 & 230 & 194 & 40 & 1.31 & 3.04 & 1.93 & 0.96 & 418 & 649 & 565 & 127 \\
\hline 3 & LS2 & 155 & 158 & 156 & 2 & 1.24 & 1.77 & 1.47 & 0.27 & 274 & 502 & 412 & 121 \\
\hline 3 & LS2-FT & 162 & 206 & 181 & 23 & 1.06 & 4.46 & 2.41 & 1.81 & 293 & 846 & 498 & 302 \\
\hline 3 & LS2-MT & 153 & 153 & 153 & 0 & 1.32 & 1.54 & 1.40 & 0.12 & 297 & 330 & 308 & 19 \\
\hline 1 & LS2-NI & 36 & 36 & 36 & NA & 0.12 & 0.12 & 0.12 & NA & 231 & 231 & 231 & NA \\
\hline 2 & LS2-TA & 162 & 164 & 163 & 2 & 1.38 & 1.52 & 1.45 & 0.10 & 227 & 264 & 246 & 26 \\
\hline 3 & LS3 & 145 & 191 & 169 & 23 & 1.38 & 1.63 & 1.52 & 0.13 & 202 & 348 & 296 & 82 \\
\hline
\end{tabular}

Differences in subject heading techniques alter both the initial and final ball dynamics and contribute to the variability found in the head responses. Future efforts could consider normalisation of the data to an average or base level for ball incoming and outgoing velocity but assumptions regarding the energy imparted by the player and subject kinematics would be required.

\section{Comparison of results relative to baseline}

To compare head response between the subjects, we reduced the data to a percentage difference compared with the baseline heading scenario (LS2). A summary of the intersubject comparisons is provided in fig 10 including average, maximum, and minimum values. A negative change corresponds to a reduction in severity compared with the baseline.

\section{DISCUSSION}

\section{Heading with varying muscle activity}

The high speed heading scenarios were expected to display different muscle activity than the low speed baseline scenario. Additional high speed heading scenarios with muscle tensing (HS3-MT) and relaxed neck muscles (HS3RLX) were also provided.

The similar peak linear and angular accelerations between the low (LS2) and high speed headings (HS3) are of interest as it would be expected that the there is a greater energy transfer to the head. The relative increase in the energy of the ball for the high speed event is actually lower than the low speed event ( $85 \% \vee 132 \%$ ). This suggests that less effort was

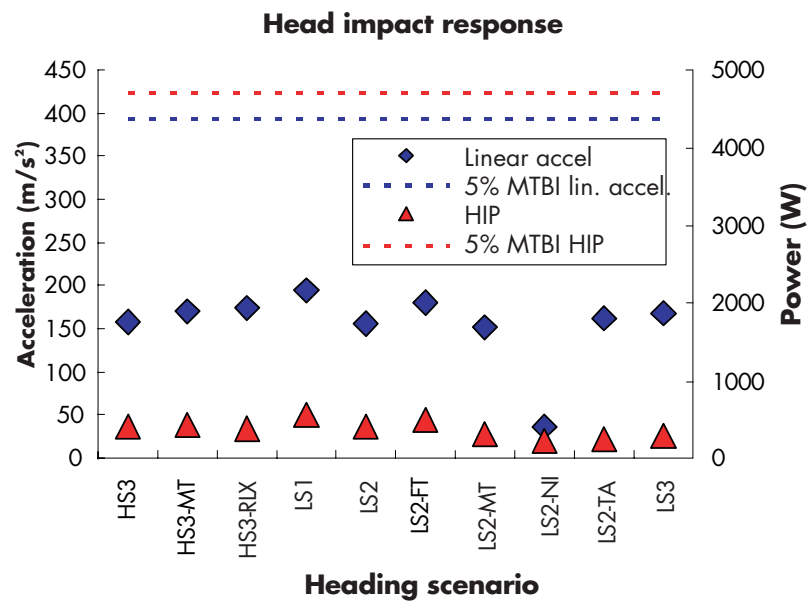

Figure 8 Composite plot of head response data by heading scenario. MTB(I), mild traumatic brain injury; see table 1 for explanation of heading codes. being expended in the high speed heading scenario. When considering the rectified $\mathrm{EMG}_{\mathrm{RMS}}$ neck muscle activity levels and duration for the high speed event, the sternocleidomastoid activity was observed to be greater across all subjects, and only small differences were noted with trapezius muscle activity. This is contrary to the reduced energy transfer and may be due to overall tensing of the active and antagonistic muscle groups trying to stabilise the head. The HIP is the same for both heading scenarios indicating a similar severity of impact.

For the pre-tensed condition (HS3-MT) the peak linear and angular accelerations increased. This suggests that the head, while being firmly held by the neck muscles, is not coupled any better to the neck or torso. As shown in the numerical study, ${ }^{40}$ pre-tensing loaded the cervical spine axially. Unexpectedly, the sternocleidomastoid muscle activity levels were lower than the normal condition. Muscle activity started earlier in the event and was sustained over a greater period of time compared with the normal case. In both conditions,
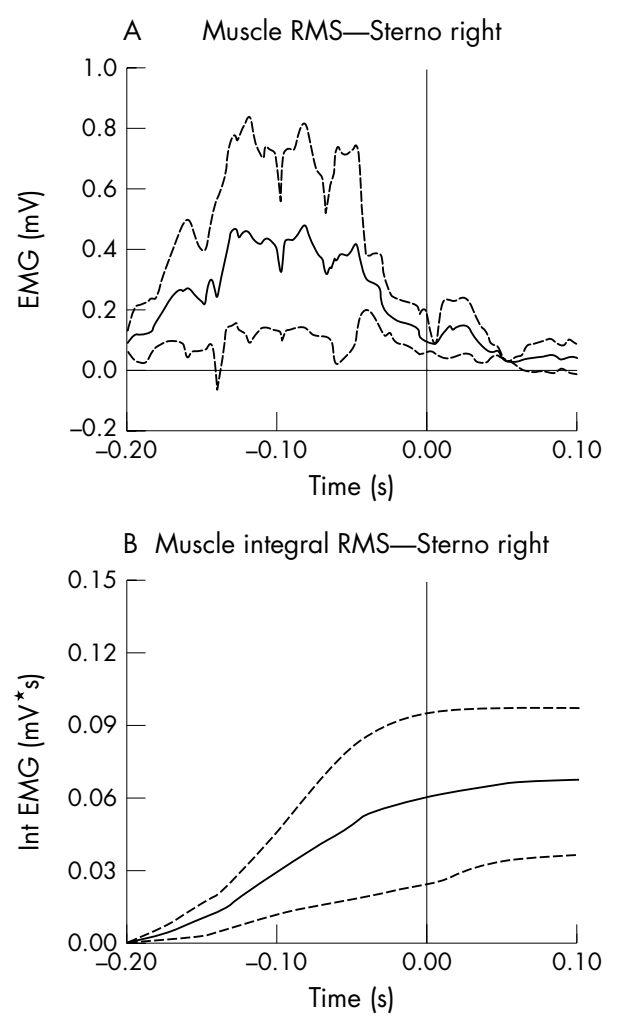

Figure 9A,B Sample neck muscle $E M G_{R M S}$ activity level and integral (mean $\pm S D$ ). RMS, root mean square. 

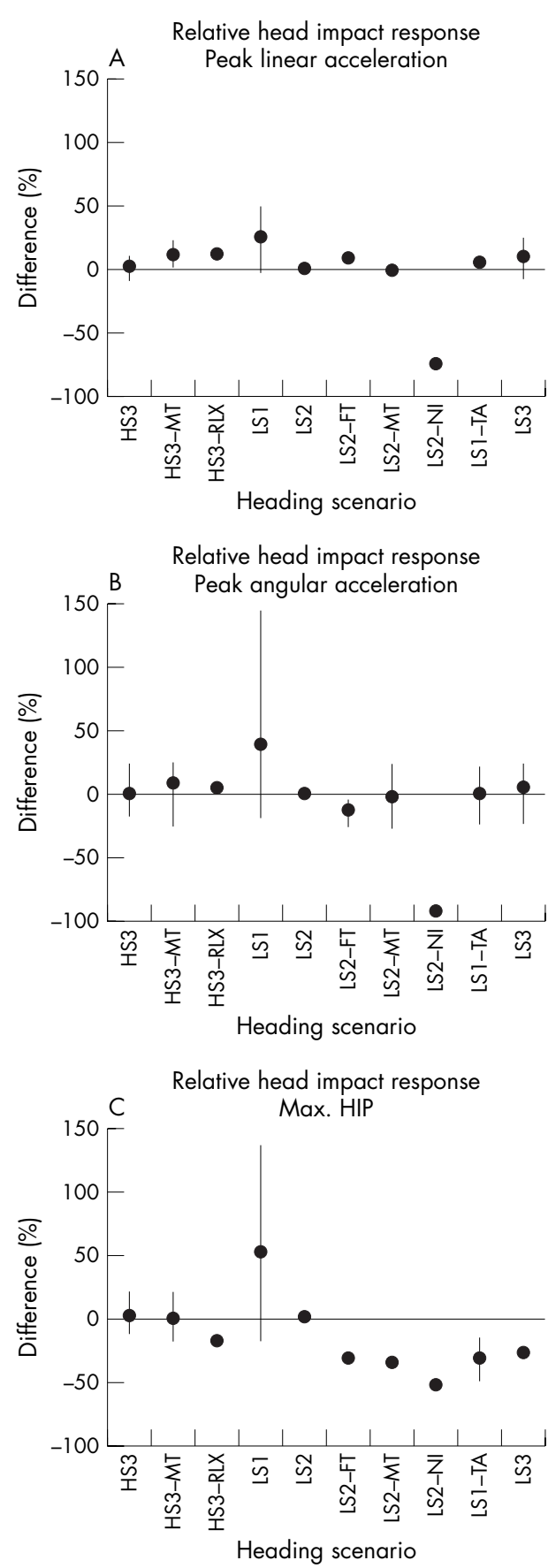

Figure 10A-C Relative differences in head impact response by heading scenario. HIP, Head Impact Power index; see table 1 for explanation of heading codes.

muscle activity reduced rapidly after impact (>50 ms). Activity levels for the trapezius muscle groups were somewhat elevated in the tensed condition.

The phasing of the head acceleration data and muscle EMG activity suggest that the neck muscles do not have time to react during the impact and provide little additional resistance. This was seen for both active and non-active headings where no increase in EMG activity was observed during the short duration of the head impact event, typically 15-20 ms.

When heading with muscles relaxed (HS3-RLX), peak linear and angular accelerations were higher compared with the baseline event. In contrast, the HIP values had decreased. The improved impact power values can be attributed to differences in head translational velocity where the head is accelerated rearwards by the ball. In contrast, the LS2 heading scenario has a greater forward head velocity prior to and after impact contributing to a greater HIP value. The EMG muscle activity levels in the relaxed condition were small with peak activity occurring after impact, presumably to re-stabilise the head.

For the low speed heading scenarios, neck muscle tensing (LS2-MT) provided a marginal reduction in peak linear/ angular head accelerations. The reduced power index suggested that muscle tensing was beneficial for low speed impacts.

\section{Heading with varying technique}

In the analysis of neck muscle activity from the initial subject trials, it was noted that sternocleidomastoid levels dropped off rapidly just prior to ball impact. A follow through scenario (LS2-FT) was therefore employed in the subject trials to extend the activation duration beyond impact. This resulted in a small increase $(7 \%)$ in peak linear acceleration and a decrease in angular acceleration $(-13 \%)$. For the same tests, the power index decreased by $33 \%$. This is likely due to small variations in body kinematics causing small changes in head accelerations and velocities. Differences in muscle activity for the follow through manoeuvre were minor.

Torso alignment was viewed as one method of reducing head response by providing a stiffer supporting structure for the head and with increased effective mass. The low speed heading scenario with torso alignment (LS2-TA) resulted in small increases $(5 \%)$ in peak linear acceleration and no difference for peak angular accelerations. The impact power was reduced $(-32 \%)$ and no significant differences in muscle EMG $\mathrm{RMS}_{\mathrm{R}}$ activity levels were observed.

The non-impact heading scenario was executed by one subject to investigate the differences in head response without ball contact. As anticipated, the peak linear/angular accelerations were different $(77 \% / 93 \%)$ from the baseline scenario. When considering HIP, the values are calculated from the anticipated time of contact to follow through. The time at which the peak HIP occurred was much later in the follow through phase $(80 \mathrm{~ms})$ compared with ball impact conditions (20 ms).

The neck muscle activity for the non-impact case leading up to point of fictitious ball contact was lower than with impact. Similar to the baseline scenario, the EMG levels for the left and right sternocleidomastoids at the time of impact were minimal in both cases.

\section{Heading with various ball targeting scenarios}

The head responses were studied for different low speed ball targeting scenarios (LS1, LS2, LS3) to achieve different body orientations, muscle activity levels, ball trajectory, head interactions, and ball exit speeds.

The head responses for the low speed heading configurations vary considerably. The LSI scenario responses (linear/ angular acceleration, HIP) were highest due to the pronounced redirection of the ball down towards the ground near the player. The kinematic data confirmed that the head velocities were higher. The neck muscle activity for the different low speed configurations did not exhibit any trend due to asymmetrical responses and fluctuating magnitudes.

The high speed heading configuration (HS3) resulted in peak head accelerations responses and power values similar to the baseline. Muscle activity levels did not differ for the muscle groups measured.

\section{Test methodology}

The current pilot study was conducted to gain a better understanding of heading biomechanics with the objective of 
identifying potential countermeasures to help reduce head impact severity. The effect of changes to heading technique were studied as a potential means of reduction since it is commonly acknowledged that proper technique is required to effectively head the ball. This may include having the proper impact location on the head, correct head-neck-torso movements, neck muscle activation, and follow through.

The methodology employed to study the effects of heading biomechanics entailed the use of physical testing and numerical simulation methods. The numerical model, with validation from initial subject tests $(n=7)$, provided the ability to study the effects of heading technique changes without the typical variability associated with human trials. Different neck muscle tensing levels and neck-torso orientations were simulated resulting in changes to the head linear and angular accelerations, and with the power experienced by the head, during contact. Although the benefits were not always consistent between the head response measures, some improvements were noted under these heading scenarios. This was used as a basis for the continuation of additional human subject trials.

A subset of the human subjects $(n=3)$ who participated in the initial trials was selected to investigate the effects of neck muscle activation and different body orientations. This was accomplished with specific standing heading scenarios requiring the subject to control and clear the ball at two different speeds $(6 \mathrm{~m} / \mathrm{s}$ and $8 \mathrm{~m} / \mathrm{s})$. By setting the target location for the ball in the different heading scenarios, it was felt that unnatural body movements were reduced and that any time required to gain familiarity with the heading scenario was also reduced. This also ensured that the findings could be directly applicable to actual game play. Additional heading scenarios were introduced by instructing the players to pre-tense their neck muscles, increase the normal follow through, and reorient their head relative to the torso with the assumption that these would vary the degree of head coupling to the torso and therefore reduce the impact severity.

Measurements obtained in the human trials included head and torso kinematics taken with high speed video, neck muscle activity through electromyography, and head response by means of an intraoral device having linear and angular accelerometers located at the mouth exterior. All measurements were taken in the midsagittal plane.

The head and torso kinematics between the initial and final subject tests were consistent in that torso rotation and rotation of the head relative to the torso were observed up to the point of contact with some subjects locking the head relative to the torso at impact. The range of rotations and the initial angles of the head and torso varied between the heading scenarios and subjects. Discernable differences in approach angle and follow through were observed for the various heading scenarios employed in the study. In general, the clearing shots placed the head in a more horizontal position and required less head rotation relative to the torso. This is in contrast to scenarios requiring greater downward ball redirection where greater head inclination and rotation is required.

Linear and angular head acceleration responses varied between the different heading scenarios and between subjects. Peak average linear resultant acceleration values in the range of $153-194 \mathrm{~m} / \mathrm{s}^{2}$ were observed and peak average angular accelerations of $1.40-2.41 \mathrm{krad} / \mathrm{s}^{2}$ were also obtained for heading scenarios involving ball impact. The values are consistent with previous research (table 6), although differences or undocumented values for ball speed, head closing speed, subject techniques, and instrumentation methods and location, as well as heading kinematics, preclude meaningful comparisons.

Large differences in head responses were observed for the heading techniques investigated in the study. This was partially attributed to different ball rebound speeds and energy transfer in the low and high speed headings. However, in some cases prominent changes in head response were not seen due to the countering effects of muscle activation levels and head orientations. The contributions of the heading scenario, ball speeds, and heading technique to the overall measured responses may obscure the effects of any one parameter change. This implies that development of generalised recommendations for heading techniques to reduce the impact severity may be problematic.

Modifications of the heading technique with either follow through or torso alignment resulted in minor increases in peak linear accelerations but were subject to large variability in responses between subjects. More significant decreases in the HIP indicated that the techniques were beneficial but again were subject to the variability in subject kinematics. Small observed changes in neck muscle activity did not indicate their involvement in the measured head responses. The implementation of the techniques will depend greatly on the circumstances surrounding the heading. Sufficient time, personal space, and player skills will influence the overall effectiveness of the manoeuvres.

The relative benefits provided by the heading scenarios (fig 10) vary depending on the selected head response measure used. In relation to peak linear accelerations, muscle tensing for the low speed baseline scenario provided some benefit but either increased or remained unchanged for the other heading scenarios. In terms of angular accelerations, the greatest benefits were seen with the follow though scenario. This is in contrast to the LS1 low speed scenario which resulted in an increase due to the increased head rotations required to redirect the ball. The HIP ranking also indicated a lesser impact severity for cases involving muscle tensing and different ball targeting locations but is inconsistent. Again, this implies that generalised recommendations to reduce impact severity will be difficult for the heading scenarios studied. Should a similar analysis also be extended to encompass all possible heading scenarios, it is expected that inconsistent results will be found.

\section{Inferences from injury measures}

The relative benefits of the heading techniques were often seen to be opposing each other depending on which injury measure was used. Increased peak acceleration responses were often paired with reductions in HIP adding confusion regarding which measure was more relevant. The relevance of the injury measures for minor or chronic impacts has not been established as the relation between the physical measurement and injury mechanism has not been defined. Caution must therefore be exercised when reviewing the results in terms of injury potential and it is more appropriate at this time to view these as measures of impact severity until such time that more low level injury data become available.

\begin{tabular}{|c|c|c|c|c|}
\hline $\begin{array}{l}\text { Ball } \\
\text { velocity } \\
(\mathrm{m} / \mathrm{s})\end{array}$ & $\begin{array}{l}\text { Peak linear } \\
\text { accel. } \\
\left(\mathrm{m} / \mathrm{s}^{2}\right)\end{array}$ & $\begin{array}{l}\text { Peak angular } \\
\text { accel. } \\
\text { (krad/s } / \mathrm{s}^{2} \text { ) }\end{array}$ & Subjects & Reference \\
\hline 9 & $158 \pm 19$ & $1.30 \pm 0.32$ & $\begin{array}{l}\text { Experienced males, } \\
n=4 \text {, responses at } \\
\text { head } C G \text {, forehead } \\
\text { impacts }\end{array}$ & $\begin{array}{l}\text { Naunheim et } \\
a f^{55}\end{array}$ \\
\hline 12 & $199 \pm 27$ & $1.46 \pm 0.30$ & & \\
\hline $\begin{array}{l}\text { Not } \\
\text { available }\end{array}$ & $\begin{array}{l}90 \text { unskilled } \\
120 \text { skilled }\end{array}$ & $0.03-0.19$ & $\begin{array}{l}\text { Recreational male, } \\
n=1 \text {, responses on } \\
\text { headband, jumping } \\
\text { forehead impacts }\end{array}$ & $\begin{array}{l}\text { Burslem and } \\
\text { Lees }^{23}\end{array}$ \\
\hline
\end{tabular}




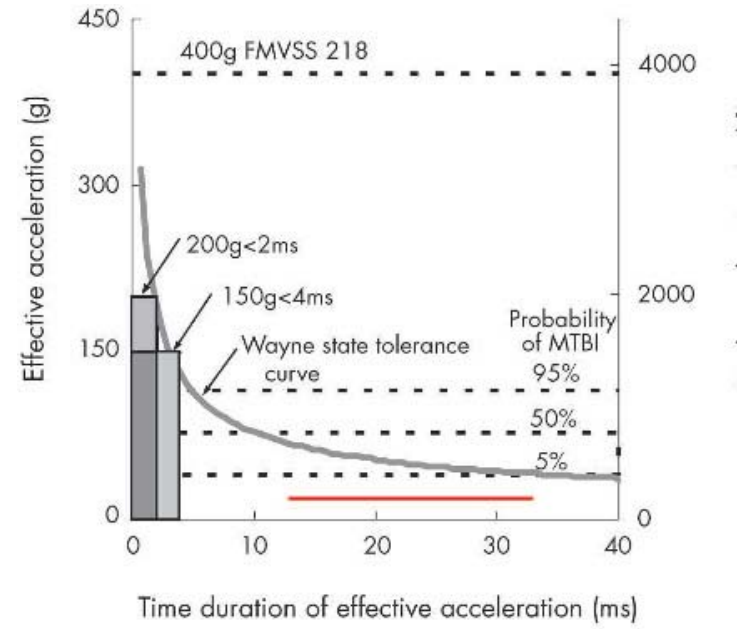

Figure 11 Linear acceleration responses (red) in relation to published tolerance values. MTBI, mild traumatic brain injury.

Furthermore, the head response measures in this study were obtained in a different manner than that used to derive the various injury indices. Accelerations reported at the centre of gravity of the head are commonly used as opposed to the mouth accelerations reported in this study.

The headings conducted in the study did not result in any mild or acute injury and are therefore suggested to be subinjurious for the limited number of impacts used in the tests. This can be confirmed qualitatively by comparing the linear accelerations with established tolerance limits for traumatic or acute brain injury, without consideration for angular kinetics. ${ }^{51}{ }^{52}$ Such a relationship is represented in the WSTC (fig 11), which illustrates an increased tolerance of the brain with shorter time durations. The curve is also the basis for common head injury criteria such as the Gadd Severity Index and Head Injury Criterion. The US Federal Motor Vehicle Safety Standard, FMVSS 218, provides time dependent tolerance limits to account for the varying tolerance. It is based on the Japan Head Tolerance Curve ${ }^{53}$ and is similar to its predecessor, the WSTC. All responses in the study were found to be below the levels required to cause acute injury.

The linear acceleration responses are also compared to the probability of MTBI. ${ }^{51}$ It can be seen that the results are well below the risk of concussion making the differences in measurement methods (mouth $v$ head centre of gravity) less pertinent. Similar findings are obtained when comparing the responses to the probability of concussion based on the HIP index (fig 12).

In terms of angular accelerations, without consideration of linear kinetics, the head responses can be compared with published tolerance limits for varying levels of brain injury. ${ }^{48} 55$ The range of responses measured in the subject tests is depicted in fig 13. Again, the responses are well below the published ranges making the differences in measurement methods less pertinent.

\section{Heading scenarios}

Although the heading configurations chosen for the pilot study represent some of the commoner scenarios, these do not necessarily reflect the full spectrum. Additional heading configurations could be studied in conjunction with their exposure in the field to establish the representative impact risks. Due to the increased complexity of three dimensional analysis required for the complete assessment of heading biomechanics, the configurations selected for the current study were simplified to two dimensional motions.

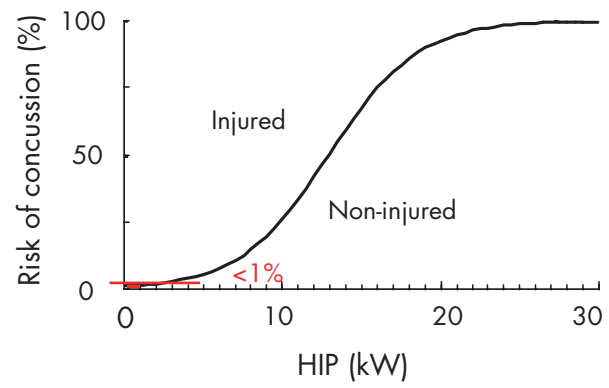

Figure 12 Measured head impact power responses (red) in relation to the risk of concussion (mild traumatic brain injury). HIP, Head Impact Power.

\section{Limitations}

The heading scenarios chosen for the study attempted to vary head-neck-torso orientations and muscle activity levels in a natural manner. Despite these efforts, the resulting heading biomechanics may not have been refined due to the limited practise time for the subjects to adapt to these new techniques. This may result in differences in head response between that seen in the study and those experienced in the field when following similar heading guidelines. Training protocols and feedback on muscle activity and resulting head response could prove valuable in realising the full potential of any recommended methods.

The heading scenarios were controlled in terms of the incoming ball speed and required heading target location. The ball rebound speed, however, was dictated by the individual technique of the player. This resulted in different amounts of energy being delivered to the ball, and hence, the corresponding head response. A means to normalise the data would have been beneficial, but this requires detailed knowledge of the kinematics, kinetics, neck loads, and mass properties. This limitation can be overcome with a greater number of subjects and trials. The use of intersubject comparisons helped reduce this limitation for the current study.

Measurement of the EMG muscle activity levels resulted in large observed variations across and within subjects. Nonetheless, the measured responses provided a qualitative assessment of timing issues while the relative magnitude was of lesser consequence. Measurement of the two major muscle groups provided an initial assessment of their involvement but the measure of additional muscle groups would provide a

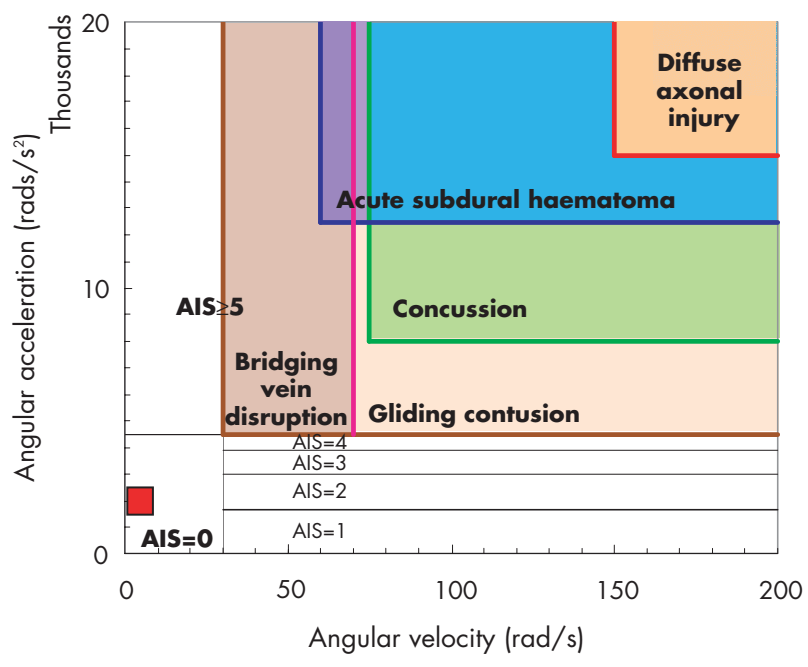

Figure 13 Measured angular acceleration responses (red) in relation to published tolerance limits for brain injury. AIS, abbreviated injury scale. 
better understanding of the overall muscle activity and application of external forces to the head for complete definition of the impact dynamics. This would also provide data for further validation and manipulation of the numerical model although effort from active and antagonistic muscle groups could prove problematic. Measure of the internal muscles will also be difficult with surface or needle EMG electrodes. Normalisation of the neck muscle EMG activity was not possible due to higher measured dynamic forces than that observed under maximal voluntary contraction. This may be due to dynamic effects, the involvement of other muscle groups during $\mathrm{MVC}$, or varying $\mathrm{EMG}$-force relations during neck movement and resulting line of action for the individual muscle groups. Alternative means, such as inverse dynamic analysis or controlled dynamic excitation trials may be required.

The peak linear acceleration measurement method for the trials exhibited good repeatability allowing for the comparison of responses across all subjects. The angular accelerations were observed to be less consistent as was the HIP index. The use of an angular accelerometer in the verification trials proved to be superior over the use of multiple linear accelerometer clusters. Nevertheless, should oscillations occur from head motion or the intraoral device, transformation of the linear accelerometer measurements to the head's centre of gravity will be difficult. More complex and expensive measurement systems using a matrix of several linear accelerometers can be developed for use in human subjects while providing three dimensional kinematic and kinetic information. External fixation of the instrumentation to the head would be required.

\section{CONCLUSION}

Heading in football is essential to the defensive and offensive strategies of the game. Modification of game rules or heading techniques will remain a remote possibility until a greater understanding of the biomechanics and injury potential is obtained. Few definitive data exist in literature demonstrating the injurious potential of ball impacts (for example mild, acute, chronic), and further research will ensure the safety of the sport. The biomechanical analysis techniques and methodology presented in this paper can provide insights into head impact response and influence of heading technique. When combined with epidemiological data, effective preventive measures can be formulated and the benefits realised.

The study successfully developed a numerical model to study the effects of heading techniques on head impact response. The model is limited to providing kinematic and kinetic data in the midsagittal plane and has been validated with a single human subject. The results of the modelling efforts pointed the way to improve heading biomechanics through changes in the neck muscle activity levels and body segment orientations. This has been demonstrated with human subject trials where kinematic, kinetic, and EMG measurements were taken.

\section{What is already known on this topic}

- Heading is an important aspect of the game of football.

- There is controversy regarding the long term consequences of heading.

- Heading technique, ball speed, and player characteristics can alter the severity of the impact to the head, but these are poorly characterised.
Some heading techniques provided benefits but were dependent on which measure of head impact response was used. Typical increases in peak linear and angular acceleration values were often countered by improvements in the HIP values. This was attributed to HIP being dependent on the duration of the accelerations and resulting velocity changes. An exception to this was the benefit provided by muscle tensing but the effects were diminished with increasing ball impact speed. When considering all possible heading configurations, conclusive recommendations for improving heading techniques cannot be made at this time.

Limitations of our study were: the variability in subject responses, the use of frontal heading scenarios, limited representation of the player population, and limited time frame for the players to become familiar with the modified techniques.

While there is concern about the injury potential with children, transfer of the current results is limited by the lower head mass, decreased neck muscle mass/strength and undeveloped heading skills. Similar differences can be noted between female and male players where females typically have lower body mass and neck musculature. Stochastic modelling methods may provide a means to represent player and environmental effects without the variability associated with subject trials. ${ }^{37}$

The implications of long term injuries from heading cannot be made due to the yet undefined injury mechanisms and tolerance levels. Preliminary comparison of the head responses in the current study demonstrated that all measures of linear and angular responses were well below tolerance limits published for TBI and MTBI.

Our results provide a number of options for the desired reduction of heading severity to the head and neck. One such alternative is to employ improved techniques such as muscle tensing or follow through. This has the benefit of reducing some aspects of the head responses while providing greater player skills for controlling the ball and perhaps greater appreciation of the game. The improved techniques can be realised through structured training and coaching but will require education of the trainers and a direct measure of the head response for immediate feedback. Use of training manuals or video would be another approach to improve heading biomechanics but if self-administered, the skill levels may not progress at the desired rate due to poor feedback. An instructional approach may provide additional benefits by educating players and coaches of potentially detrimental practices. Public availability of the information can also be beneficial to educate the general population, governing bodies, health professionals, and even parents, of

\section{What this study adds}

- Biomechanical methods for analysing head response to ball impact was developed and include both kinematic/kinetic measurements and neck muscle EMG methods.

- A numerical model of the player was validated and implemented for comparative heading analysis.

- Variations in heading technique were investigated with empirical and analytical methods and their influence on head impact response documented.

- A qualitative assessment of injury risk was made in relation to mild and traumatic brain injury criteria.

- The consequences of repeated impacts in heading remain to be quantified. 
the safety aspects of the game. The actual injury risk level of headings in the short or long term remains to be established.

In summary, the present study provided a greater understanding of heading biomechanics. A number of recommendations related to heading techniques were identified but will require careful consideration. Inconsistent results drawn from the modified heading techniques will make this a difficult approach to justify. Alternative countermeasures may include changes to the ball characteristics as this has been shown to provide on overall benefit and one that can be effectively implemented. ${ }^{41}$ An enhanced study with a larger sample, greater variety of heading scenarios and more comprehensive measurement capability will aid in the further understanding and improvement of heading biomechanics. ${ }^{23}$

\section{ACKNOWLEDGEMENTS}

The research has been supported through the contributions of the FIFA Medical Assessment and Research Centre (F-MARC). The authors wish to acknowledge the participation of the football teams, the Gloucester Hornets and Kanata Soccer (Ontario, Canada) for participation of their players as test subjects.

\section{Authors' affiliations}

N Shewchenko, C Withnall, M Keown, R Gittens, Biokinetics and Associates Ltd, Ontario, Canada

J Dvorak, FIFA Medical Assessment and Research Centre, Zurich, Switzerland

Competing interests: none declared

\section{REFERENCES}

1 Dvorak J, Junge A. Football injuries and physical symptoms. A review of the literature. Am Orthop Soc Sports Med 2000;28:S3-S9.

2 Boden B, Kirkendall D, Garrett W. Concussion incidence in elite college soccer players. Am J Sports Med 1998;26:238-41.

3 Barnes B, Cooper L, Kirkendall D, et al. Concussion history in elite male and female soccer players. Am J Sports Med 1998:26:433-8.

4 Matser J, Kessels A, Lezak M, et al. Neuropsychological impairment in amateur soccer players. JAMA 1999;282:971-3.

5 Tysvaer A. Head and neck injuries in soccer, impact of minor trauma. Sports Med 1992;14:200-13.

6 Peterson L, Junge A, Chomiak J, et al. Incidence of football injuries and complaints in different age groups and skill-level groups. Am J Sports Med 2000;28:S-51-S-57.

7 Tysvaer A, Lochen E. Soccer injuries to the brain. A neuropsychologic study of former soccer players. Am J Sports Med 1991;19:56-60.

8 Tysvaer A, Storli O, Bachen N. Soccer injuries to the brain. A neurologic and electroencephalographic study of former players. Acta Neurol 1989;80:151-6.

9 McCrory P. Brain injury and heading in soccer. BMJ 2003;327:351-2.

10 Anderson S, Griesemet B, Johnson M, et al. Injuries in youth soccer: a subject review. Pediatrics 2000;105:659-61.

11 Matser J, Kessels A, Jordan B, et al. Chronic traumatic brain injury in professional soccer players. Am Acad Neurol 1998;51:791-6.

12 Matser J, Kessels A, Lezak M, et al. A dose-response relation of headers and concussions with cognitive impairment in professional soccer players. J Clin Exp Neuropsychol 2001; 23:770-4.

13 Kirkendall D, Garrett W. Heading in soccer: integral skill or grounds for cognitive dysfunction? J Athl Train 2001;36:328-33.

14 Janda D, Bir C, Cheney A. An evaluation of the cumulative effect of soccer heading in the youth population. Inj Control Saf Promot 2002;9:25-31.

15 Guskiewicz K, Marshall S, Broglio S, et al. No evidence of impaired neurocognitive performance in collegiate soccer players. Am J Sports Med 2002;30:157-62

16 Green G, Jordan S. Are brain injuries a significant problem in soccer? Clin Sports Med 1998;17:795-809.

17 Jordan S, Green G, Galanty H, et al. Acute and chronic brain injury in United States national team soccer players. Am J Sports Med 1996;24:205-10.

18 Putukian M, Echemendia R, Mackin S. The acute neuropsychological effects of heading in soccer: a pilot study. Clin J Sport Med 2000;10:104-9.

19 Johnston K, Lassonde M, Ptito A. A contemporary neurosurgical approach to sport-related head injury: The McGill Concussion Protocol. Am Coll Surg 2001;192:515-23.

20 Johnston K, McCrory P, Mohtadi G, et al. Evidence-based review of sportrelated concussion: clinical science. Clin J Sport Med 2001;11:150-9.

21 Andersen T, Arnason A, Engebretsen L, et al. Mechanisms of head injuries in elite football. Br J Sports Med 2004;38:690-6.

22 Sortland O, Tysvaer A, Storli O. Changes in cervical spine in association football players. Br J Sports Med 1982;16:80-4.

23 Burslem I, Lees A. Quantification of ball impact accelerations of the head during the heading of a football. In: Reilly T, Lees A, Davids K, Murphy WJ, eds. Science and Football. Liverpool: E \& FN Spon, 1988:243-8.
24 Ludwig KM. A Biomechanical Analysis of Soccer Heading Technique. Denton: Texas Women's University, 1998

25 Naunheim R, Bayly P, Standeven J, et al. Linear and angular head accelerations during heading a soccer ball. Med Sci Sports Exerc 2003:35:1406-12.

26 Schneider K, Zernicke R. Computer simulation of head impact: estimation of head-injury risk during soccer heading. Int I Sport Biomech 1988;4:258-371.

27 Bayly P, Naunheim R, Standeven J, et al. Linear and angular accelerations of the human head during heading of a soccer ball. Second Joint EMBS/BMES Conference, 23 October 2002, Houston, TX, pp. 2577-8.

28 Lewis L, Naunheim R, Standeven J, et al. Do football helmets reduce acceleration of impact in blunt head injuries. Acad Emerg Med $2001: 8: 604-9$.

29 Naunheim R, Ryden A, Standeven J, et al. Does soccer headgear attenuate the impact when heading a soccer ball? Acad Emerg Med 2003;10:85-90.

30 Hardy W. Instrumentation in experimental design. In: Nahum A, Melvin J, eds. Accidental Injury-Biomechanics and Prevention, 2nd edn. New York: Springer-Verlag, 2002:27-9.

31 Bauer JA, Thomas TS, Cauraugh JH, et al. Impact forces and neck muscle activity in heading by collegiate female soccer players. I Sports Sci 2001; 19:171-9.

32 Gurdjian ES, Lissner MS, Patrick MS, et al. Intracranial pressure and accelerations accompanying head impacts in human cadavers. Surg Gynecol Obstet 1961;113:185-90.

33 Mawdsley HP. A biomechanical analysis of heading. Momentum 1978;3:30-40.

34 Lynch JM, Baver J. Heading. In: Garrett W, Kirkendall D, Contiguglia SR, eds. The US Soccer Sports Med Book. Baltimore, MD: Williams \& Wilkins, 1996:81-85.

35 Klein GN, Mannion AF, Panjabi MM, et al. Trapped in the neutral zone: another symptom of whiplash-associated disorder. Eur Spine J 2001;10:141-8.

36 Szabo TJ, Welcher JB. Human subject kinematics and electromyographic activity during low speed rear impacts. 40th Stapp Car Crash Conference, Society of Automotive Engineers, 1996, Alberqueque, NM.

37 Babbs CF. Biomechanics of heading a soccer ball: implications for player safety. West Lafayette, IN: Purdue University, 8 August, 2001.

38 Ziejewski $M$, Swenson R, Schanfield P, et al. A biomechanical examination of the efficacy of soccer protective headgear in reducing trauma to the head from low impacts. The Brain Injury Association's 20th Annual Symposium, 2003, Atlanta, GA

39 Queen RM, Weinhold PS, Kirkendall DT, et al. Theoretical study of ball properties on impact force on soccer heading. Med Sci Sports Med 2003:35:2069-76

40 Shewchenko N, Withnall C, Keown M, et al. Heading in football. Part 2 Biomechanics of ball heading and head response. Br J Sports Med 2005;39(suppl I):i26-i32.

41 Shewchenko N, Withnall C, Keown M, et al. Heading in football. Part 3: Effect of ball properties and head response. Br J Sports Med 2005;39/suppl I): $: 33-i 39$.

42 Moss S, Wang Z, Salloum M, et al. Anthropometry for WorldSID. A world harmonized midsize male side impact crash dummy. Government/Industry Meeting, June 2000, Washington, DC.

43 Tortora GJ, Anagnostakos NP (eds). Principles of Anatomy and Physiology, 4th edn., New York: Harper \& Row 1984.

44 Newman J, Shewchenko N, Beusenberg M, et al. Verification of biomechanical methods employed in a comprehensive study of mild traumatic brain injury and the effectiveness of american football helmets. J Biomech 2005, (in press).

45 Gurdjian ES, Webster JE, Lissner HR. Observations on the mechanism of brain concussion. Surg Gynecol Obstet 1955;101:680-90.

46 Holbourne AHS. Mechanics of head injuries. Lancet 1943;245:438-41.

47 Hirsch AE, Ommaya AK. Protection from brain injury: the relative significance of translational and rotational motions of the head after impact. 14th Stapp Car Crash Conference, Society of Automotive Engineers, 1970, Ann Arbor, $\mathrm{Ml}$.

48 Ommaya AK. Head injury biomechanics. Association for the Advancement of Automotive Medicine, 1984. Denver, CO, 1984

49 Newman J. A generalized acceleration model for brain injury threshold (GAMBIT). International Research Council on the Biomechanics of Impact (IRCOBI), 2-4 September 1986, Zurich, Switzerland.

50 Kramer F, Appel H. Evaluation of protection criteria on the basis of statistical biomechanics. IRCOBI Conference on the Biomechanics of Impacts, 1990, Lyon, France.

51 Newman J, Shewchenko N, Welbourne E. A proposed new biomechanical head injury index assessment function-the maximum power index. 44th Stapp Car Crash Conference, SAE International, 6-8 November 2000, Atlanta, GA.

52 Gurdjian ES, Thomas L. Tolerance curves of acceleration and intracranial pressure and protective index in experimental head injury. J Trauma 1966;6:600-4

53 Ono I, Kikuchi A, Nakamura M, et al. Human head tolerance to sagittal impact reliable estimation deduced from experimental head injury using subhuman primates and cadaver skulls. 24th Stapp Car Crash Conference, SAE International 1980, Troy MI.

54 Thibault L, Gennarelli T. Brain injury: an analysis of neural and neurovascular trauma in the nonhuman primate. 34th Annual Conference of the Association for the Advancement of Automotive Medicine, 1990, Scottsville, AZ.

55 Glaister D. Acceleration injury. Scientific Foundations of Trauma. Jordan Hill, Oxford: Butterworth-Heinemann, 1997:314-23. 